Supporting information

\title{
$\beta$-Acyloxysulfonyl Tethers for Intramolecular Diels-Alder Cycloaddition Reactions
}

\author{
Nataliya Chumachenko, ${ }^{*, \dagger}$ Paul Sampson, ${ }^{*, \dagger}$ Allen D. Hunter ${ }^{\ddagger}$ \\ and Matthias Zeller ${ }^{\ddagger}$ \\ Department of Chemistry, Kent State University, Kent, Ohio 44242, and \\ Department of Chemistry, Youngstown State University, One University Plaza, \\ Youngstown, Ohio 44555 \\ psampson@kent.edu
}

\section{General}

\section{Experimental}

Unless otherwise indicated, ${ }^{1} \mathrm{H}$ NMR spectra were recorded on a Bruker AMX 300 spectrometer at $300 \mathrm{MHz}$, using TMS as an internal reference. ${ }^{13} \mathrm{C}$ NMR spectra were recorded on the same spectrometer at $75 \mathrm{MHz}$, with the $\mathrm{CDCl}_{3}$ as an internal reference. Indicated ${ }^{1} \mathrm{H}$ NMR spectra were recorded on a Bruker AVANCE $400 \mathrm{NMR}$ spectrometer at $400 \mathrm{MHz}$. NOE spectra were recorded on the same spectrometer at $400 \mathrm{MHz}$ or a Varian $500 \mathrm{NMR}$ spectrometer at $500 \mathrm{MHz}$. TLC analysis was performed using silica coated plates (Sorbent Technologies). Flash column chromatography was conducted on silica gel Premium Rf Grade (40-75 $\mu \mathrm{m}$ (200x400 mesh), Sorbent Technologies). The glass pressure vessel $(150 \mathrm{~mL})$ was purchased from Chemglass and used with magnetic stirring. Commercial reagents and solvents (Acros) (including anhydrous $\mathrm{CH}_{2} \mathrm{Cl}_{2}$ ) were used as received. THF was freshly distilled from $\mathrm{Na} /$ benzophenone. Deionized water was used for aqueous reactions. All non-aqueous reactions were carried out under an atmosphere of argon using vacuo- or oven-dried glassware, employing syringe techniques. Commercially available $2.5 \mathrm{M} n$-BuLi solution in hexane (Aldrich) was used. Melting points were determined using a Thomas-Hoover apparatus and are uncorrected.

Attention: In the absence of solvent, compounds 3-10 and 14a-i were prone to facile polymerization. They could be stored without noticeable decomposition for up to a week as dichloromethane solutions at $-5^{\circ} \mathrm{C}$. When preparing these compounds, the removal of solvents after column chromatography was always performed in vacuo at $0-5{ }^{\circ} \mathrm{C}$; the oily residue was immediately diluted with dichloromethane and stored in the presence of 2,6-di-tert-butyl-4methylphenol at $-5^{\circ} \mathrm{C}$. As a result, it was impractical to obtain combustion analysis data for these compounds.

The yields of compounds 3-10 and 14a-i were calculated by ${ }^{1} \mathrm{H}$ NMR analysis using 2,6-di-tertbutyl-4-methylphenol as an internal standard. To evaluate the reliability of these calculations, in several cases the solvent was removed in high vacuo and the isolated yield was compared with that established by use of an internal standard. These yields were always in good agreement (no more than $1 \%$ deviation).

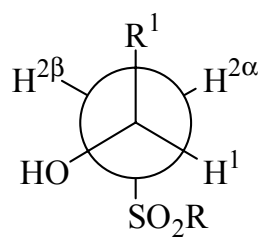

$\mathbf{A}$

Figure S1

${ }^{\dagger}$ Kent State University

* Youngstown State University (X-ray crystallography) 
The assignment of protons $\mathrm{H}^{2 \alpha}$ and $\mathrm{H}^{2 \beta}$ in the various $\beta$-hydroxy sulfone (4,7-10) and $\beta$-acyloxy sulfone products (14a-h) were made assuming that, in $\mathrm{CDCl}_{3}$ solution, $\mathbf{A}$ (Figure $\mathrm{S} 1, \mathrm{R}=\mathrm{C}_{4} \mathrm{H}_{5}$ ) is the preferred conformation. The protons $\mathrm{H}^{2 \alpha}, \mathrm{H}^{2 \beta}$ and $\mathrm{H}^{1}$ compose a first-order AMX system if $\Delta v / \mathrm{J}_{\mathrm{AB}}>6$, or a non-first-order $\mathrm{ABX}$ system if $\Delta v / \mathrm{J}_{\mathrm{AB}}<6$. For the ABX system, only $\mathrm{J}_{\mathrm{AB}}$ values below can be considered as an accurate measure of the coupling constant; apparent values of $\mathrm{J}_{\mathrm{AX}}$ and $\mathrm{J}_{\mathrm{BX}}$, presented in the text below, are dependent on spectrometer frequency. ${ }^{*}$ The $\Delta \mathrm{v} /$ $\mathrm{J}_{\mathrm{AB}}$ values for each compound are presented in Table $\mathrm{S} 1$.

Table $\mathrm{S} 1 . \Delta \boldsymbol{v} / \mathrm{J}_{\mathrm{AB}}$ values for $\beta$-hydroxy sulfones 4,7-10 and $\beta$-acyloxy sulfones $\mathbf{1 4 \mathbf { b } - \mathbf { h }}$

\begin{tabular}{|l|l|l|l|l|l|l|l|l|l|l|l|l|}
\hline Comp. & $\mathbf{4}$ & $\mathbf{7}$ & $\mathbf{8}$ & $\mathbf{9}$ & $\mathbf{1 0}$ & $\mathbf{1 4 b}$ & $\mathbf{1 4 c}$ & $\mathbf{1 4 d}$ & $\mathbf{1 4 e}$ & $\mathbf{1 4 f}$ & $\mathbf{1 4 g}$ & $\mathbf{1 4 h}$ \\
\hline$\Delta \mathrm{v} / \mathrm{J}_{\mathrm{AB}}$ & 1.7 & 4.3 & 2.7 & 4.1 & 2.3 & 5.3 & 7.2 & $<1$ & 5.1 & 7.2 & 5.2 & 8.2 \\
\hline
\end{tabular}

\section{Preparation of $\beta$-hydroxy sulfones 3,4 and 7-10}

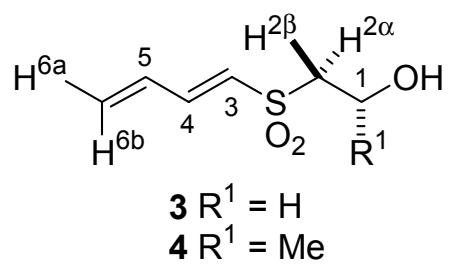

\subsection{2-[(E)-Butadienesulfonyl]ethanol (3).}

Butadiene sulfone (1) (4.73 g, $40.0 \mathrm{mmol}, 1$ equiv) was dissolved in anhydrous THF (120 mL) under argon, and was cooled in a dry ice/acetone bath. $n$-BuLi $(2.5 \mathrm{M}$ in hexanes, $16.0 \mathrm{~mL}, 40.0$ mmol, 1.00 equiv) was added dropwise over $25 \mathrm{~min}$, maintaining the reaction temperature below $-68{ }^{\circ} \mathrm{C}$. Initially, a yellow solution was observed, and then a cream precipitate formed. The addition of $n$-BuLi was stopped after a bright red coloration had developed in the reaction mixture. The reaction mixture was allowed to warm up to $-50{ }^{\circ} \mathrm{C}$, and then water $(30 \mathrm{~mL})$ and hydroquinone (ca $0.05 \mathrm{~g}$ ) were added. The precipitate immediately dissolved, and the red coloration disappeared. The organic solvents were removed in vacuo, resulting in a light yellow aqueous solution (ca $30 \mathrm{~mL}$ ) of $\mathrm{Li}(Z)$-1,4-butadienylsulfinate. This solution was added dropwise, with stirring and cooling in an ice-water bath, to a $150 \mathrm{~mL}$ glass pressure vessel containing $\mathrm{ZnCl}_{2}$ (2.67-2.83 g, 19.6-20.8 mmol, 0.49-0.52 equiv) in water ( $\left.20 \mathrm{~mL}\right)$. A white precipitate of zinc (Z)-1,4-butadienylsulfinate formed. An additional aliquot of water $(40 \mathrm{~mL})$ was used to complete the transfer of the Li sulfinate. Ethylene oxide $(6.00 \mathrm{~mL}, 5.29 \mathrm{~g}, 120$ mmol, 3.00 equiv) was added; the vessel was closed and kept at $70-75^{\circ} \mathrm{C}$ with stirring for $3 \mathrm{~h}$. The resulting reaction mixture was cooled to rt, filtered and the precipitate was washed with water $(3 \times 20 \mathrm{~mL})$. The combined aqueous fractions were extracted with dichloromethane $(4 \times 100$ $\mathrm{mL})$. The combined dichloromethane extracts (ca $400 \mathrm{~mL}$ ) were treated with 2,5-di-tert-butyl-4methylphenol (ca $0.20 \mathrm{~g}$ ), dried over $\mathrm{MgSO}_{4}$, filtered and concentrated in vacuo at $0-5{ }^{\circ} \mathrm{C}$ to a volume of approx. $150 \mathrm{~mL}$ and treated with DMAP ( $0.500 \mathrm{~g}, 4.09 \mathrm{mmol}, 0.10$ equiv). After $36 \mathrm{~h}$ at $\mathrm{rt}$, the resulting reaction mixture was concentrated in vacuo and immediately subjected to column chromatography (EtOAc/Hexanes, 1.5/1) to afford the title compound 3 (1.97 g, 12.1 mmol, $30 \%, \mathrm{R}_{\mathrm{f}}=0.24$ ) as a light yellow oil.

${ }^{1} \mathrm{H}$ NMR $\left(\mathrm{CDCl}_{3}\right) \delta 3.00(\mathrm{t}, \mathrm{J}=6.0 \mathrm{~Hz}, 1 \mathrm{H}, \mathrm{OH}), 3.30-3.25\left(\mathrm{~m}, 2 \mathrm{H}, \mathrm{H}^{2}\right), 4.09-4.03\left(\mathrm{~m}, 2 \mathrm{H}, \mathrm{H}^{1}\right)$, 5.68 (br d, J=10.0 Hz, 1H, $\mathrm{H}^{6 \mathrm{a}}$ ), 5.77 (br d, J=16.9 Hz, 1H, $\mathrm{H}^{6 \mathrm{~b}}$ ), $6.43(\mathrm{dd}, \mathrm{J}=14.9,0.6 \mathrm{~Hz}$. 1H, $\mathrm{H}^{3}$ ), 6.48 (dddd, J=16.6, 10.9, 10.0, 0.6 Hz, $1 \mathrm{H}, \mathrm{H}^{5}$ ), $7.21\left(\mathrm{br} \mathrm{dd}, \mathrm{J}=15.0,10.8 \mathrm{~Hz}, 1 \mathrm{H}, \mathrm{H}^{4}\right) ;{ }^{13} \mathrm{C}$ $\mathrm{NMR}\left(\mathrm{CDCl}_{3}\right.$, one signal masked) $\delta 56.4,57.4,129.0,132.5,144.3$.

\footnotetext{
* Crews, P.; Rodríguez, J.; Jaspars, M. Organic Structure Analysis, Oxford University Press, New York Oxford, 1998, pp 111-128.
} 
1.2. 1-[(E)-Butadienesulfonyl]propan-2-ol (4).

The title compound $4\left(2.13 \mathrm{~g}, 12.1 \mathrm{mmol}, 30 \%, \mathrm{R}_{\mathrm{f}}(\right.$ EtOAc/Hexanes, $\left.1.5 / 1)=0.30\right)$ was prepared as light yellow oil by procedure 1.1, employing propylene oxide $(8.00 \mathrm{~mL}, 7.06 \mathrm{~g}, 122 \mathrm{mmol}$, 3.05 equiv) instead of ethylene oxide.

${ }^{1} \mathrm{H} \mathrm{NMR}\left(\mathrm{CDCl}_{3}\right) \delta 1.31\left(\mathrm{~d}, \mathrm{~J}=6.4 \mathrm{~Hz}, 3 \mathrm{H}, \mathrm{CH}_{3}\right), 3.21(\mathrm{~d}, \mathrm{~J}=2.8 \mathrm{~Hz}, 1 \mathrm{H}, \mathrm{OH}), 3.09$ (dd, J=14.4, $\left.2.8 \mathrm{~Hz}, 1 \mathrm{H}, \mathrm{H}^{2 \alpha}\right), 3.17\left(\mathrm{dd}, \mathrm{J}=14.5,8.7 \mathrm{~Hz}, 1 \mathrm{H}, \mathrm{H}^{2 \beta}\right), 4.39\left(\right.$ app dqt, $\mathrm{J}=9.0,6.3,2.8 \mathrm{~Hz}, 1 \mathrm{H}, \mathrm{H}^{1}$ ), 5.69 (br d, J=10.0 Hz, 1H, H $\mathrm{H}^{6 \mathrm{a}}$ ), 5.77 (br d, J=16.7 Hz, 1H, $\mathrm{H}^{\mathrm{b}}$ ), 6.43 (br d, J=15.0 Hz. 1H, $\mathrm{H}^{3}$ ), 6.47 (dddd, $\left.\mathrm{J}=16.9,10.8,10.0,0.5 \mathrm{~Hz}, 1 \mathrm{H}, \mathrm{H}^{5}\right), 7.22\left(\mathrm{br} \mathrm{dd}, \mathrm{J}=15.0,10.9 \mathrm{~Hz}, 1 \mathrm{H}, \mathrm{H}^{4}\right) ;{ }^{13} \mathrm{C} \mathrm{NMR}$ $\left(\mathrm{CDCl}_{3}\right) \delta 22.9,62.4,62.7,128.7,129.4,132.4,144.8$.<smiles>[R]C(=O)CS(=O)CC([R])=O</smiles>

\subsection{2-[(Z)-Butadienesulfonyl]-1-phenylethanone (5).}

Butadiene sulfone (1) (3.55 g, $30.0 \mathrm{mmol}, 1.00$ equiv) was dissolved in anhydrous THF (60 mL) under argon and cooled in a dry ice/acetone bath. $n$-BuLi (2.5M in hexanes, $12.0 \mathrm{~mL}, 30.0$ mmol, 1.00 equiv) was added dropwise over $15 \mathrm{~min}$, maintaining the reaction temperature below $-68{ }^{\circ} \mathrm{C}$. Initially, a yellow solution was observed, and then a cream precipitate formed. The addition of $n$-BuLi was stopped immediately after a bright red coloration had developed in the reaction mixture liquid phase. After just 2-3 min of stirring at $-78^{\circ} \mathrm{C}$, trimethylchlorosilane $(4.10 \mathrm{~mL}, 3.49 \mathrm{~g}, 32.1 \mathrm{mmol}, 1.07$ equiv) was added dropwise. Little or no rise in the reaction temperature was observed. After the first drops of TMSCl had been added, the reaction mixture liquid phase became light yellowish, while the precipitate did not change in color (cream). After stirring the resulting mixture at $-78{ }^{\circ} \mathrm{C}$ for $25 \mathrm{~min}$, the precipitate disappeared. The mixture was stirred for a further $1 \mathrm{~h}$ at $-78^{\circ} \mathrm{C}$. A solution of $\alpha$-bromoacetophenone $(15.7 \mathrm{~g}, 78.9 \mathrm{mmol}, 2.63$ equiv) in THF ( $40 \mathrm{~mL})$ was then added dropwise over $15 \mathrm{~min}$, followed by $\mathrm{Bu}_{4} \mathrm{NF}(32 \mathrm{~mL}, 1 \mathrm{M}$ solution in THF, $32 \mathrm{mmol}, 1.07$ equiv). It is important to add the ketone slowly because it partially precipitates out of the cold reaction mixture. Both additions proceeded without noticeable increases in the reaction temperature. The reaction mixture was left in the dry ice/acetone bath to warm up to rt overnight.

The reaction mixture was concentrated in vacuo. The oily residue was twice co-evaporated with EtOAc $(150 \mathrm{~mL})$ in vacuo. A third addition of EtOAc $(150 \mathrm{~mL})$ resulted in the formation of a white precipitate of $\mathrm{Bu}_{4} \mathrm{NBr}$. After 15 min of stirring at $\mathrm{rt}$, the precipitate was filtered out and the filtrate was concentrated in vacuo. Column chromatography was performed with gradient elution: the first elution (EtOAc/Hexane, 1/10) gave recovered $\alpha$-bromoacetophenone; the second elution (EtOAc/Hexane, $1 / 3 ; \mathrm{R}_{\mathrm{f}}$ of $\mathbf{5}=0.20, \mathrm{R}_{\mathrm{f}}$ of $\alpha$-bromoacetophenone $\left.=0.55\right)$ gave the title compound 5 as a colorless oil (4.98 g, $21.1 \mathrm{mmol}, 70 \%)$.

${ }^{1} \mathrm{H}$ NMR $\left(\mathrm{CDCl}_{3}\right) \delta 4.68\left(\mathrm{~s}, 2 \mathrm{H}, \mathrm{CH}_{2}\right), 5.64\left(\mathrm{br} \mathrm{d}, \mathrm{J} \sim 11 \mathrm{~Hz}, 1 \mathrm{H}, \mathrm{H}^{6 \mathrm{a}}\right),{ }^{\neq} 5.65$ (br d, J 16 Hz, 1H, $\left.\mathrm{H}^{6 \mathrm{~b}}\right),{ }^{\neq} 6.29$ (br d, J=11.1 Hz, 1H, $\left.\mathrm{H}^{3}\right), 6.76$ (app t, J=11.3 Hz, 1H, $\mathrm{H}^{4}$ ); 7.38 (dddd, J=17.2, 11.5, 9.6, 1.0 Hz, $\left.1 \mathrm{H}, \mathrm{H}^{5}\right), 7.48-7.55(\mathrm{~m}, 2 \mathrm{H}, \mathrm{Ph}), 7.61-7.68(\mathrm{~m}, 1 \mathrm{H}, \mathrm{Ph}), 7.97-8.02(\mathrm{~m}, 2 \mathrm{H}, \mathrm{Ph}) ;{ }^{13} \mathrm{C}$ $\operatorname{NMR}\left(\mathrm{CDCl}_{3}\right) \delta 63.2,125.7,129.1,129.5,129.9,130.0,134.7,135.9,144.3,188.7$.

\subsection{1-[(Z)-Butadienesulfonyl]-3,3-dimethyl-2-butanone (6).}

The title compound $6(0.867 \mathrm{~g}, 4.01 \mathrm{mmol}, 40 \%)$ was obtained as colorless oil by procedure 1.3 from butadiene sulfone (1) $(1.18 \mathrm{~g}, 10.0 \mathrm{mmol}, 1.00$ equiv) in anhydrous THF (20 mL) using trimethylchlorosilane (1.35 mL, $1.15 \mathrm{~g}, 10.6 \mathrm{mmol}, 1.06$ equiv), 1-bromo-3,3-dimethyl-2-

\footnotetext{
${ }^{\neq}$Exact magnitude of the coupling constant is difficult to calculate because of the signal broadness.
} 
butanone (3.60 mL, 4.47 g. $25.0 \mathrm{mmol}, 2.50$ equiv) in anhydrous THF ( $5 \mathrm{~mL})$ and $\mathrm{Bu}_{4} \mathrm{NF}(10.6$ $\mathrm{mL}, 1 \mathrm{M}$ solution in THF, $10.6 \mathrm{mmol}, 1.06$ equiv).

${ }^{1} \mathrm{H} \mathrm{NMR}\left(\mathrm{CDCl}_{3}\right) \delta 1.19(\mathrm{~s}, 9 \mathrm{H}, t-\mathrm{Bu}), 4.25\left(\mathrm{~s}, 2 \mathrm{H}, \mathrm{CH}_{2}\right), 5.65\left(\mathrm{br} \mathrm{d}, \mathrm{J} \sim 10 \mathrm{~Hz}, 1 \mathrm{H}, \mathrm{H}^{6 \mathrm{a}}\right),{ }^{\neq} 5.64(\mathrm{br}$ d, J 17 Hz, 1H, $\mathrm{H}^{6 \mathrm{~b}}$ ), ${ }^{\neq} 6.35$ (br d, J=11.1 Hz, 1H, $\left.\mathrm{H}^{3}\right), 6.72\left(\right.$ app t, J=11.3 Hz, 1H, $\left.\mathrm{H}^{4}\right), 7.35-7.49$ $\left(\mathrm{m}, 1 \mathrm{H}, \mathrm{H}^{5}\right) ;{ }^{13} \mathrm{C} \mathrm{NMR}\left(\mathrm{CDCl}_{3}\right) \delta 25.8,45.4,61.2,126.6,129.5,130.2,143.3,204.2$.

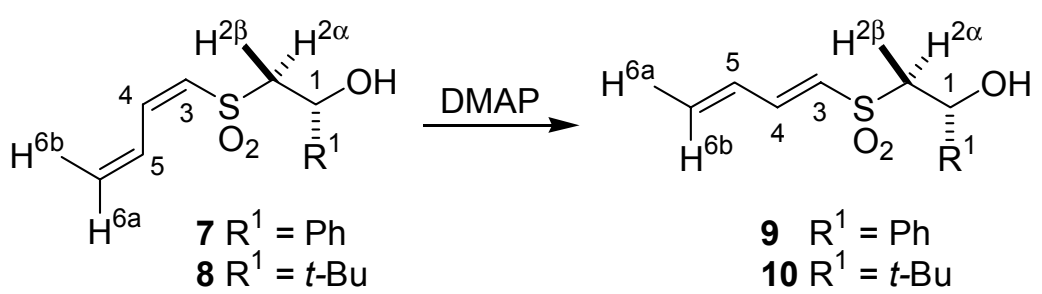

\subsection{2-[(Z)-Butadienesulfonyl]-1-phenylethanol (7).}

$\alpha$-Sulfonyl ketone 5 (4.61 g, $19.5 \mathrm{mmol}, 1.00$ equiv) was dissolved in methanol (240 mL) and cooled to $-10{ }^{\circ} \mathrm{C}$. Sodium borohydride $(0.863 \mathrm{~g}, 22.8 \mathrm{mmol}, 1.17$ equiv) was dissolved in water $(10 \mathrm{~mL})$ at $-10{ }^{\circ} \mathrm{C}$ and was added dropwise to the solution of $\alpha$-sulfonyl ketone 5 with stirring, so that the temperature never rose above $-7^{\circ} \mathrm{C}$. The resulting reaction mixture was stirred at -5 ${ }^{\circ} \mathrm{C}$ for an additional $1 \mathrm{~h} 15 \mathrm{~min}$, and was then treated with EtOAc $(240 \mathrm{~mL})$ and water $(200 \mathrm{~mL})$. The solvents (ca $200 \mathrm{~mL}$ ) were removed in vacuo. Additional ethyl acetate $(200 \mathrm{~mL})$ was added and, again, the solvents (ca $250 \mathrm{~mL}$ ) were removed in vacuo. The residue was treated with EtOAc $(200 \mathrm{~mL})$, which resulted in its separation into two layers. The organic layer was washed with water $(50 \mathrm{~mL})$. The combined aqueous layers were extracted with dichloromethane $(3 \times 50$ $\mathrm{mL})$. The combined organic layers were then dried with $\mathrm{MgSO}_{4}$, filtered and concentrated in vacuo to afford a crude oil, which was used directly in the next step without purification. The title compound could be purified by column chromatography (EtOAc/Hexane, 1/3, $\mathrm{R}_{\mathrm{f}}=0.24$ ), which afforded 7 as a colorless oil $(4.29 \mathrm{~g}, 18.0 \mathrm{mmol}, 92 \%)$.

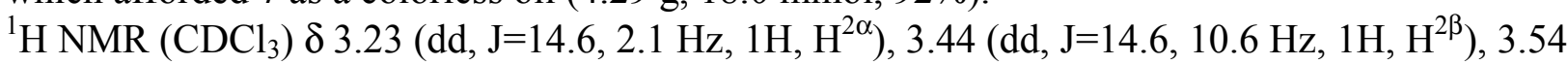
$(\mathrm{d}, \mathrm{J}=2.7 \mathrm{~Hz}, 1 \mathrm{H}, \mathrm{OH}), 5.31$ (app dt, J=10.1, 2.4 Hz, 1H, $\left.\mathrm{H}^{1}\right), 5.65\left(\right.$ br d, J 17 Hz, 1H, $\mathrm{H}^{6 \mathrm{~b}}$ ), 5.67 (br d, J 10 Hz, 1H, H ${ }^{6 a}$ ), 6.19 (br d, J=11.1 Hz, 1H, $\mathrm{H}^{3}$ ), 6.71 (app t, J=11.3 Hz, 1H, H ${ }^{4}$ ), 7.37-7.51 (m, $\left.1 \mathrm{H}, \mathrm{H}^{5}\right), 7.26-7.37(\mathrm{~m}, 5 \mathrm{H}, \mathrm{Ph}) ;{ }^{13} \mathrm{C} \mathrm{NMR}\left(\mathrm{CDCl}_{3}\right) \delta$ 63.8, 68.9, 125.9, 126.6, 128.6, 129.0, 129.7, 130.0, 141.1, 143.7.

\subsection{2- $[(E)$-Butadienesulfonyl]-1-phenylethanol (9).}

Crude $\beta$-hydroxysulfone 7 , obtained by procedure 1.5 after concentration in vacuo, was diluted with dichloromethane $(100 \mathrm{~mL})$, treated with DMAP $(0.464 \mathrm{~g}, 3.80 \mathrm{mmol}, 0.20$ equiv) and left for two days at rt. The reaction mixture was concentrated in vacuo and immediately subjected to column chromatography (EtOAc/Hexane, 1/3, $\left.\mathrm{R}_{\mathrm{f}}=0.24\right)$ to afford the title compound 9 as a colorless oil (4.29 g, $18.0 \mathrm{mmol}, 92 \%$ for two steps from 5).

${ }^{1} \mathrm{H}$ NMR $\left(\mathrm{CDCl}_{3}\right) \delta 3.18\left(\mathrm{dd}, \mathrm{J}=14.7,2.4 \mathrm{~Hz}, 1 \mathrm{H}, \mathrm{H}^{2 \alpha}\right), 3.38\left(\mathrm{dd}, \mathrm{J}=14.6,10.0 \mathrm{~Hz}, 1 \mathrm{H}, \mathrm{H}^{2 \beta}\right), 3.71$ $(\mathrm{d}, \mathrm{J}=3.0 \mathrm{~Hz}, 1 \mathrm{H}, \mathrm{OH}), 5.23\left(\mathrm{app} d \mathrm{~J}=10.0,2.6 \mathrm{~Hz}, 1 \mathrm{H}, \mathrm{H}^{1}\right), 5.63\left(\right.$ br d, J=10.0 Hz, $\left.1 \mathrm{H}, \mathrm{H}^{6 \mathrm{a}}\right)$, 5.71 (br d, J=16.9 Hz, 1H, H $\left.{ }^{6 b}\right), 6.41\left(\right.$ app dt, J=16.9, $\left.10.4 \mathrm{~Hz}, 1 \mathrm{H}, \mathrm{H}^{5}\right), 6.44(\mathrm{~d}, \mathrm{~J}=15.3 \mathrm{~Hz}, 1 \mathrm{H}$, $\left.\mathrm{H}^{3}\right), 7.15\left(\mathrm{dd}, \mathrm{J}=14.9,11.0 \mathrm{~Hz}, 1 \mathrm{H}, \mathrm{H}^{4}\right), 7.23-7.38(\mathrm{~m}, 5 \mathrm{H}, \mathrm{Ph}) ;{ }^{13} \mathrm{C} \mathrm{NMR}\left(\mathrm{CDCl}_{3}\right) \delta$ 62.9, 69.0, 126.0, 128.5, 128.97, 129.02, 129.4, 132.6, 141.3, 144.2.

\subsection{1- $[(Z)$-Butadienesulfonyl]-3,3-dimethyl-2-butanol (8).}

The title compound $8(0.732 \mathrm{~g}, 3.35 \mathrm{mmol}, 85 \%)$ was obtained as a colorless oil by procedure 1.5 from $\alpha$-sulfonyl ketone 6 ( $0.852 \mathrm{~g}, 3.94 \mathrm{mmol}, 1.00$ equiv) in methanol (125 mL) and sodium borohydride $(0.175 \mathrm{~g}, 4.63 \mathrm{mmol}, 1.17$ equiv) in water $(4 \mathrm{~mL})$.

${ }^{1} \mathrm{H}$ NMR $\left(\mathrm{CDCl}_{3}\right) \delta 0.93\left(\mathrm{~s}, 9 \mathrm{H}, t\right.$-Bu), $3.08\left(\mathrm{dd}, \mathrm{J}=14.3,9.9 \mathrm{~Hz}, 1 \mathrm{H}, \mathrm{H}^{2 \beta}\right), 3.09(\mathrm{~d}, \mathrm{~J}=3.2 \mathrm{~Hz}, 1 \mathrm{H}$, $\mathrm{OH}), 3.21\left(\mathrm{dd}, \mathrm{J}=14.3,1.5 \mathrm{~Hz}, 1 \mathrm{H}, \mathrm{H}^{2 \alpha}\right), 3.88\left(\mathrm{ddd}, \mathrm{J}=9.9,3.0,1.5 \mathrm{~Hz}, 1 \mathrm{H}, \mathrm{H}^{1}\right.$ ), 5.67 (br d, J 17 
$\left.\mathrm{Hz}, 1 \mathrm{H}, \mathrm{H}^{6 \mathrm{~b}}\right),{ }^{\neq} 5.68\left(\right.$ br d, J $\left.\sim 9 \mathrm{~Hz}, 1 \mathrm{H}, \mathrm{H}^{6 \mathrm{a}}\right),{ }^{\neq} 6.20\left(\mathrm{br} \mathrm{d}, \mathrm{J}=11.1 \mathrm{~Hz}, 1 \mathrm{H}, \mathrm{H}^{3}\right), 6.74$ (app t, J=11.3

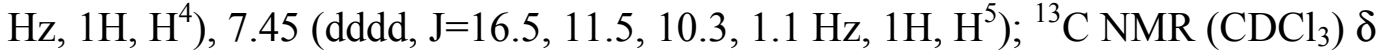
$25.5,35.1,58.6,73.6,126.5,129.6,129.9,143.6$.

\subsection{1-[(E)-Butadienesulfonyl]-3,3-dimethyl-2-butanol (10).}

The title compound $10(0.732 \mathrm{~g}, 3.35 \mathrm{mmol}, 85 \%)$ was obtained as a colorless oil by procedure 1.6 from the crude $\beta$-hydroxy sulfone 8 , using DMAP ( $0.092 \mathrm{~g}, 0.75 \mathrm{mmol}, 0.22$ equiv) in $\mathrm{CH}_{2} \mathrm{Cl}_{2}(50 \mathrm{~mL})$ for 4 days at $\mathrm{rt}$.

${ }^{1} \mathrm{H} \mathrm{NMR}\left(\mathrm{CDCl}_{3}\right) \delta 0.93(\mathrm{~s}, 9 \mathrm{H}, t-\mathrm{Bu}), 3.08\left(\mathrm{dd}, \mathrm{J}=14.4,9.9 \mathrm{~Hz}, 1 \mathrm{H}, \mathrm{H}^{2 \beta}\right), 3.19(\mathrm{dd}, \mathrm{J}=14.4,1.7$ $\left.\mathrm{Hz}, 1 \mathrm{H}, \mathrm{H}^{2 \alpha}\right), 3.86\left(\mathrm{dd}, \mathrm{J}=9.9,1.7 \mathrm{~Hz}, 1 \mathrm{H}, \mathrm{H}^{1}\right), 5.66\left(\mathrm{br} \mathrm{d}, \mathrm{J}=10.4 \mathrm{~Hz}, 1 \mathrm{H}, \mathrm{H}^{6 \mathrm{a}}\right), 5.75($ br d, $\left.\mathrm{J}=16.9 \mathrm{~Hz}, 1 \mathrm{H}, \mathrm{H}^{6 \mathrm{~b}}\right), 6.40-6.54\left(\mathrm{~m}, 1 \mathrm{H}, \mathrm{H}^{5}\right), 6.52\left(\mathrm{~d}, \mathrm{~J}=15.1 \mathrm{~Hz}, 1 \mathrm{H}, \mathrm{H}^{3}\right), 7.15(\mathrm{br} d \mathrm{~d}, \mathrm{~J}=15.0$, $\left.10.9 \mathrm{~Hz}, 1 \mathrm{H}, \mathrm{H}^{4}\right) ;{ }^{13} \mathrm{C}$ NMR $\left(\mathrm{CDCl}_{3}\right) \delta 25.6,35.2,57.9,73.6,128.9,129.3,132.5,144.0$.

\section{Acylation of $\beta$-hydroxy sulfones $3,4,9$ and 10 with $\alpha, \beta$-unsaturated acids: Preparation of $\beta$-acyloxy sulfones 14a-i.}

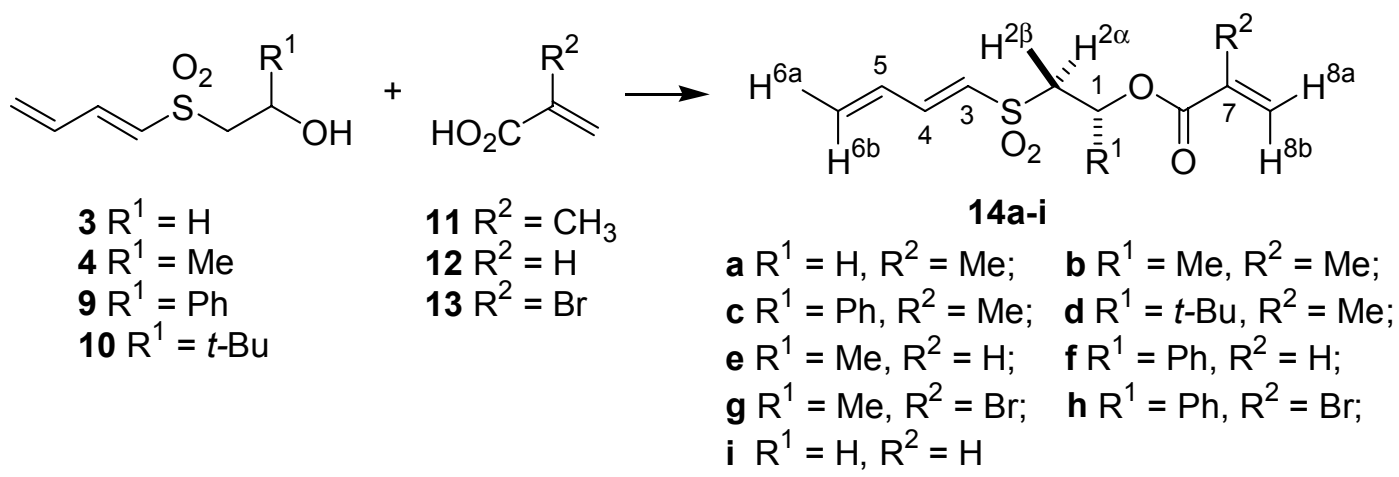

\subsection{2-[(E)-Butadienesulfonyl]ethyl methacrylate (14a).}

A solution of DCC (1.74 g, $8.43 \mathrm{mmol}, 1.41$ equiv) in anhydrous dichloromethane $(3 \mathrm{~mL})$ was added dropwise over $25 \mathrm{~min}$ at $\mathrm{rt}$ to a stirred solution of $\beta$-hydroxy sulfone $3(0.973 \mathrm{~g}, 6.00$ mmol, 1.00 equiv), methacrylic acid (11) $(0.77 \mathrm{~mL}, 0.78 \mathrm{~g}, 9.1 \mathrm{mmol}$. 1.5 equiv) and DMAP $(0.147 \mathrm{~g}, 1.20 \mathrm{mmol}, 0.20$ equiv) in anhydrous dichloromethane $(8 \mathrm{~mL})$. A white precipitate formed. The reaction mixture was stirred for $24 \mathrm{~h}$ at $\mathrm{rt}$ and then filtered. The filtered precipitate was washed with dichloromethane and dried in air to afford the recovered dicyclohexyl urea (DCU) byproduct $(1.50 \mathrm{~g}, 6.69 \mathrm{mmol})$. The combined dichloromethane filtrate was concentrated in vacuo and immediately subjected to column chromatography (EtOAc/Hexane, $1 / 2, \mathrm{R}_{\mathrm{f}}=0.27$ ) to give the title compound 14a as a colorless oil (1.18 g, $5.12 \mathrm{mmol}, 85 \%)$.

${ }^{1} \mathrm{H} \mathrm{NMR}\left(\mathrm{CDCl}_{3}\right) \delta 1.90\left(\operatorname{app~t}, \mathrm{J}=1.3 \mathrm{~Hz}, 3 \mathrm{H}, \mathrm{CH}_{3}\right), 3.38\left(\mathrm{t}, \mathrm{J}=5.9 \mathrm{~Hz}, 2 \mathrm{H}, \mathrm{H}^{2}\right), 4.52(\mathrm{t}, \mathrm{J}=5.9$ $\mathrm{Hz}, 2 \mathrm{H}, \mathrm{H}^{1}$ ), 5.59 (quintet, $\mathrm{J}=1.5 \mathrm{~Hz}, 1 \mathrm{H}, \mathrm{H}^{8 \mathrm{a}}$ ), $5.64\left(\mathrm{br} \mathrm{d}, \mathrm{J}=10.1 \mathrm{~Hz}, 1 \mathrm{H}, \mathrm{H}^{6 \mathrm{a}}\right.$ ), 5.73 (br d, J=16.9 $\left.\mathrm{Hz}, 1 \mathrm{H}, \mathrm{H}^{6 \mathrm{~b}}\right), 6.08\left(\mathrm{br} \mathrm{s}, 1 \mathrm{H}, \mathrm{H}^{8 \mathrm{~b}}\right), 6.39$ (dd, J=15.0, $\left.0.5 \mathrm{~Hz}, 1 \mathrm{H}, \mathrm{H}^{3}\right), 6.41$ (dddd, $\mathrm{J}=16.9,10.9$, 10.0, 0.6 Hz, $\left.1 \mathrm{H}, \mathrm{H}^{5}\right), 7.17\left(\mathrm{dd}, \mathrm{J}=15.0,10.9 \mathrm{~Hz}, 1 \mathrm{H}, \mathrm{H}^{4}\right) ;{ }^{13} \mathrm{C} \mathrm{NMR}\left(\mathrm{CDCl}_{3}\right) \delta 18.3,54.3,58.2$, $126.8,128.9,129.3,132.3,135.6,144.8,166.7$.

\subsection{1-[(E)-Butadienesulfonyl]prop-2-yl methacrylate (14b).}

The title compound 14b (93\%) was obtained by procedure 1.9 from $\beta$-hydroxy sulfone 4 after column chromatography (EtOAc/Hexane, $1 / 2.5, \mathrm{R}_{\mathrm{f}}=0.29$ ).

${ }^{1} \mathrm{H} \mathrm{NMR}\left(\mathrm{CDCl}_{3}\right) \delta 1.43\left(\mathrm{~d}, \mathrm{~J}=6.4 \mathrm{~Hz}, 3 \mathrm{H}, \mathrm{CH}_{3}\right), 1.92\left(\right.$ app t, J=1.3 Hz, 3H, $\left.\mathrm{CH}_{3}\right), 3.20(\mathrm{dd}$, $\left.\mathrm{J}=14.8,4.2 \mathrm{~Hz}, 1 \mathrm{H}, \mathrm{H}^{2 \alpha}\right), 3.46\left(\mathrm{dd}, \mathrm{J}=14.8,7.8 \mathrm{~Hz}, 1 \mathrm{H}, \mathrm{H}^{2 \beta}\right), 5.41(\mathrm{ddq}, \mathrm{J}=7.8,4.2,6.4 \mathrm{~Hz}, 1 \mathrm{H}$, $\mathrm{H}^{1}$ ), 5.60 (quintet, $\mathrm{J}=1.6 \mathrm{~Hz}, 1 \mathrm{H}, \mathrm{H}^{8 \mathrm{a}}$ ), 5.65 (br d, $\mathrm{J}=9.8 \mathrm{~Hz}, 1 \mathrm{H}, \mathrm{H}^{6 \mathrm{a}}$ ), 5.74 (br d, J=16.8 Hz, $1 \mathrm{H}$, $\left.\mathrm{H}^{6 \mathrm{~b}}\right), 6.10\left(\mathrm{br} \mathrm{s}, 1 \mathrm{H}, \mathrm{H}^{8 \mathrm{~b}}\right), 6.38\left(\mathrm{~d}, \mathrm{~J}=15.1 \mathrm{~Hz}, 1 \mathrm{H}, \mathrm{H}^{3}\right), 6.42\left(\operatorname{app~dt}, \mathrm{J}=16.9,10.4 \mathrm{~Hz}, 1 \mathrm{H}, \mathrm{H}^{5}\right)$, 
$7.15\left(\mathrm{dd}, \mathrm{J}=14.9,11.0 \mathrm{~Hz}, 1 \mathrm{H}, \mathrm{H}^{4}\right) ;{ }^{13} \mathrm{C} \mathrm{NMR}\left(\mathrm{CDCl}_{3}\right) \delta 18.3,20.3,60.0,65.8,126.5,128.8$, $129.1,132.3,136.0,144.6,166.1$.

\subsection{2- $[(E)$-Butadienesulfonyl]-1-phenylethyl methacrylate (14c).}

The title compound $\mathbf{1 4 c}(81 \%)$ was obtained by procedure 1.9 from $\beta$-hydroxy sulfone 9 after column chromatography $\left(\mathrm{CH}_{2} \mathrm{Cl}_{2} /\right.$ Hexane, $\left.3 / 1, \mathrm{R}_{\mathrm{f}}=0.13\right)$.

${ }^{1} \mathrm{H}$ NMR $\left(\mathrm{CDCl}_{3}\right) \delta 1.92\left(\right.$ br s, $\left.3 \mathrm{H}, \mathrm{CH}_{3}\right), 3.39\left(\mathrm{dd}, \mathrm{J}=15.0,3.3 \mathrm{~Hz}, 1 \mathrm{H}, \mathrm{H}^{2 \alpha}\right), 3.75(\mathrm{dd}, \mathrm{J}=15.0$, $\left.9.6 \mathrm{~Hz}, 1 \mathrm{H}, \mathrm{H}^{2 \beta}\right), 5.60-5.66\left(\mathrm{~m}, 2 \mathrm{H}, \mathrm{H}^{8 \mathrm{a}}, \mathrm{H}^{6 \mathrm{a}}\right), 5.72\left(\mathrm{br} \mathrm{d}, \mathrm{J}=16.9 \mathrm{~Hz}, 1 \mathrm{H}, \mathrm{H}^{6 \mathrm{~b}}\right), 6.17(\mathrm{br} \mathrm{s}, 1 \mathrm{H}$, $\left.\mathrm{H}^{8 \mathrm{~b}}\right), 6.26\left(\mathrm{br} \mathrm{d}, \mathrm{J}=14.9 \mathrm{~Hz}, 1 \mathrm{H}, \mathrm{H}^{3}\right), 6.30\left(\mathrm{dd}, \mathrm{J}=9.6,3.3 \mathrm{~Hz}, 1 \mathrm{H}, \mathrm{H}^{1}\right), 6.36($ app dt, $\mathrm{J}=16.9,10.6$ $\left.\mathrm{Hz}, 1 \mathrm{H}, \mathrm{H}^{5}\right), 7.14\left(\mathrm{dd}, \mathrm{J}=14.9,10.9 \mathrm{~Hz}, 1 \mathrm{H}, \mathrm{H}^{4}\right), 7.27-7.43(\mathrm{~m}, 5 \mathrm{H}, \mathrm{Ph}) ;{ }^{1} \mathrm{H}$ NMR $\left(\mathrm{CD}_{3} \mathrm{OD}\right) \delta$ $1.92\left(\mathrm{dd}, \mathrm{J}=1.6,1.0 \mathrm{~Hz}, 3 \mathrm{H}, \mathrm{CH}_{3}\right), 3.52\left(\mathrm{dd}, \mathrm{J}=15.0,3.0 \mathrm{~Hz}, 1 \mathrm{H}, \mathrm{H}^{2 \alpha}\right), 3.96(\mathrm{dd}, \mathrm{J}=15.0,9.9 \mathrm{~Hz}$, $\left.1 \mathrm{H}, \mathrm{H}^{2 \beta}\right), 5.63\left(\mathrm{br} \mathrm{d}, \mathrm{J}=10.1 \mathrm{~Hz}, 1 \mathrm{H}, \mathrm{H}^{6 \mathrm{a}}\right), 5.67\left(\mathrm{~m}, 1 \mathrm{H}, \mathrm{H}^{8 \mathrm{a}}\right), 5.75\left(\mathrm{br} \mathrm{d}, \mathrm{J}=16.9 \mathrm{~Hz}, 1 \mathrm{H}, \mathrm{H}^{6 \mathrm{~b}}\right)$, 6.18 (br s, $1 \mathrm{H}, \mathrm{H}^{8 \mathrm{~b}}$ ), $6.26\left(\mathrm{dd}, \mathrm{J}=9.9,3.0 \mathrm{~Hz}, 1 \mathrm{H}, \mathrm{H}^{1}\right), 6.49$ (ddd, $\mathrm{J}=16.9,10.8,10.0,0.4 \mathrm{~Hz}, 1 \mathrm{H}$, $\left.\mathrm{H}^{5}\right), 6.57\left(\mathrm{dd}, \mathrm{J}=14.9,0.6 \mathrm{~Hz}, 1 \mathrm{H}, \mathrm{H}^{3}\right), 7.14\left(\mathrm{br} \mathrm{dd}, \mathrm{J}=14.9,10.9 \mathrm{~Hz}, 1 \mathrm{H}, \mathrm{H}^{4}\right), 7.29-7.45(\mathrm{~m}, 5 \mathrm{H}$, $\mathrm{Ph}) ;{ }^{13} \mathrm{C} \mathrm{NMR}\left(\mathrm{CDCl}_{3}\right) \delta 18.4,60.8,70.7,126.6,126.8,128.8,129.2,129.2,132.3,135.9,137.9$, 144.8, 165.6.

\subsection{1-[(E)-Butadienesulfonyl]-3,3-dimethylbut-2-yl methacrylate (14d).}

A solution of DCC $(1.06 \mathrm{~g}, 5.14 \mathrm{mmol}, 1.30$ equiv) in anhydrous dichloromethane $(3 \mathrm{~mL})$ was added dropwise using a syringe pump over $1 \mathrm{~h}$ with stirring to a solution of methacrylic acid (11) $(0.85 \mathrm{~mL}, 0.86 \mathrm{~g}, 10 \mathrm{mmol}, 2.54$ equiv) in anhydrous dichloromethane $(3 \mathrm{~mL})$. After stirring for an additional $3 \mathrm{~h}$, the reaction mixture was filtered under argon and the filtrate was added dropwise over $25 \mathrm{~min}$ to a solution of $\beta$-hydroxy sulfone $\mathbf{1 0}(0.852 \mathrm{~g}, 3.94 \mathrm{mmol}, 1$ equiv) and DMAP ( $0.635 \mathrm{~g}, 5.20 \mathrm{mmol}, 1.32$ equiv) in anhydrous dichloromethane $(5 \mathrm{~mL})$. The reaction mixture was stirred at $\mathrm{rt}$ for $15 \mathrm{~h}$, filtered, and the filtrate was concentrated in vacuo and immediately subjected to column chromatography to give the title compound $\mathbf{1 4 d}$ as a colorless oil $(0.567 \mathrm{~g}, 1.98 \mathrm{mmol}, 50 \%)$.

${ }^{1} \mathrm{H}$ NMR $\left(\mathrm{CDCl}_{3}\right) \delta 0.94(\mathrm{~s}, 9 \mathrm{H}, t-\mathrm{Bu}), 1.93\left(\operatorname{app~t}, \mathrm{J}=1.2 \mathrm{~Hz}, 3 \mathrm{H}, \mathrm{CH}_{3}\right), 3.27\left(\mathrm{~m}, 2 \mathrm{H}, \mathrm{H}^{2 \alpha, 2 \beta}\right)$, $5.32\left(\mathrm{~m}, 1 \mathrm{H}, \mathrm{H}^{1}\right), 5.59\left(\right.$ app quintet, $\left.\mathrm{J}=1.5 \mathrm{~Hz}, 1 \mathrm{H}, \mathrm{H}^{8 \mathrm{a}}\right), 5.63\left(\mathrm{br} \mathrm{d}, \mathrm{J}=10.0 \mathrm{~Hz}, 1 \mathrm{H}, \mathrm{H}^{6 \mathrm{a}}\right), 5.74(\mathrm{br}$ $\left.\mathrm{d}, \mathrm{J}=16.8 \mathrm{~Hz}, 1 \mathrm{H}, \mathrm{H}^{6 \mathrm{~b}}\right), 6.11\left(\right.$ br s, $\left.1 \mathrm{H}, \mathrm{H}^{8 \mathrm{~b}}\right), 6.34\left(\mathrm{br} \mathrm{d}, \mathrm{J}=14.9 \mathrm{~Hz}, 1 \mathrm{H}, \mathrm{H}^{3}\right), 6.39$ (app dt, J=16.9, $\left.10.5 \mathrm{~Hz}, 1 \mathrm{H}, \mathrm{H}^{5}\right), 7.13\left(\mathrm{dd}, \mathrm{J}=14.9,10.9 \mathrm{~Hz}, 1 \mathrm{H}, \mathrm{H}^{4}\right) ;{ }^{13} \mathrm{C} \mathrm{NMR}\left(\mathrm{CDCl}_{3}\right.$, one signal masked $) \delta$ $18.5,25.7,35.6,56.2,74.1,126.3,128.1,129.0,132.4,136.0,145.1,166.3$.

\subsection{1-[(E)-Butadienesulfonyl]prop-2-yl acrylate (14e).}

The title compound $14 \mathrm{e}(83 \%)$ was obtained by procedure 1.9 from $\beta$-hydroxy sulfone 4 and acrylic acid (12) after column chromatography (EtOAc/Hexane, 1/2.5, $\mathrm{R}_{\mathrm{f}}=0.24$ ).

${ }^{1} \mathrm{H}$ NMR $\left(\mathrm{CDCl}_{3}\right) \delta 1.44\left(\mathrm{~d}, \mathrm{~J}=6.4 \mathrm{~Hz}, 3 \mathrm{H}, \mathrm{CH}_{3}\right), 3.19\left(\mathrm{dd}, \mathrm{J}=14.8,4.4 \mathrm{~Hz}, 1 \mathrm{H}, \mathrm{H}^{2 \alpha}\right), 3.44(\mathrm{dd}$, $\left.\mathrm{J}=14.8,7.6 \mathrm{~Hz}, 1 \mathrm{H}, \mathrm{H}^{2 \beta}\right), 5.42\left(\mathrm{ddq}, \mathrm{J}=7.6,4.4,6.4 \mathrm{~Hz}, 1 \mathrm{H}, \mathrm{H}^{1}\right), 5.66\left(\mathrm{br} \mathrm{d}, \mathrm{J}=9.9 \mathrm{~Hz}, 1 \mathrm{H}, \mathrm{H}^{6 \mathrm{a}}\right)$, $5.74\left(\right.$ br d, $\left.\mathrm{J}=16.9 \mathrm{~Hz}, 1 \mathrm{H}, \mathrm{H}^{6 \mathrm{~b}}\right), 5.87\left(\mathrm{dd}, \mathrm{J}=10.4,1.5 \mathrm{~Hz}, 1 \mathrm{H}, \mathrm{H}^{8 \mathrm{a}}\right), 6.06(\mathrm{dd}, \mathrm{J}=17.2,10.4 \mathrm{~Hz}$, $\left.1 \mathrm{H}, \mathrm{H}^{7}\right), 6.37\left(\mathrm{dd}, \mathrm{J}=14.9,0.6 \mathrm{~Hz}, 1 \mathrm{H}, \mathrm{H}^{3}\right), 6.41\left(\mathrm{dd}, \mathrm{J}=17.2,1.5 \mathrm{~Hz}, 1 \mathrm{H}, \mathrm{H}^{8 \mathrm{~b}}\right), 6.42$ (dddd, $\left.\mathrm{J}=16.9,10.9,10.0,0.6 \mathrm{~Hz}, 1 \mathrm{H}, \mathrm{H}^{5}\right), 7.16\left(\mathrm{ddt}, \mathrm{J}=14.9,10.9,0.7 \mathrm{~Hz}, 1 \mathrm{H}, \mathrm{H}^{4}\right) ;{ }^{13} \mathrm{C} \mathrm{NMR}\left(\mathrm{CDCl}_{3}\right)$ $\delta 20.3,60.0,65.7,128.1,128.8,129.2,131.9,132.4,144.7,164.9$.

\subsection{2-[(E)-Butadienesulfonyl]-1-phenylethyl acrylate (14f).}

The title compound $\mathbf{1 4 f}(81 \%)$ was obtained by procedure 1.12 from $\beta$-hydroxy sulfone 9 and acrylic acid (12) after column chromatography $\left(\mathrm{CH}_{2} \mathrm{Cl}_{2} /\right.$ Hexane, $\left.3 / 1, \mathrm{R}_{\mathrm{f}}=0.11\right)$.

${ }^{1} \mathrm{H}$ NMR $\left(\mathrm{CDCl}_{3}\right) \delta 3.39\left(\mathrm{dd}, \mathrm{J}=15.0,3.5 \mathrm{~Hz}, 1 \mathrm{H}, \mathrm{H}^{2 \alpha}\right), 3.75\left(\mathrm{dd}, \mathrm{J}=15.0,9.5 \mathrm{~Hz}, 1 \mathrm{H}, \mathrm{H}^{2 \beta}\right), 5.64$ (br d, J=10.0 Hz, 1H, H ${ }^{6 \mathrm{a}}$ ), 5.71 (br d, J=16.9 Hz, 1H, $\mathrm{H}^{6 \mathrm{~b}}$ ), 5.87 (dd, J=10.4, 1.4 Hz, 1H, H ${ }^{8 \mathrm{a}}$ ), $6.10\left(\mathrm{dd}, \mathrm{J}=17.3,10.4 \mathrm{~Hz}, 1 \mathrm{H}, \mathrm{H}^{7}\right), 6.26\left(\mathrm{br} \mathrm{d}, \mathrm{J}=14.9 \mathrm{~Hz}, 1 \mathrm{H}, \mathrm{H}^{3}\right), 6.32(\mathrm{dd}, \mathrm{J}=9.5,3.4 \mathrm{~Hz}, 1 \mathrm{H}$, $\left.\mathrm{H}^{1}\right), 6.36\left(\right.$ app dt, $\left.\mathrm{J}=16.9,10.4 \mathrm{~Hz}, 1 \mathrm{H}, \mathrm{H}^{5}\right), 6.43\left(\mathrm{dd}, \mathrm{J}=17.3,1.4 \mathrm{~Hz}, 1 \mathrm{H}, \mathrm{H}^{8 b}\right), 7.13(\mathrm{dd}, \mathrm{J}=14.9$, 
$\left.10.9 \mathrm{~Hz}, 1 \mathrm{H}, \mathrm{H}^{4}\right), 7.27-7.43(\mathrm{~m}, 5 \mathrm{H}, \mathrm{Ph}) ;{ }^{13} \mathrm{C} \mathrm{NMR}\left(\mathrm{CDCl}_{3}\right.$, one signal masked) $\delta 60.5,70.6$, $126.7,127.9,128.8,129.16,129.24,132.3,132.4,137.7,144.8,164.4$.

\subsection{1-[(E)-Butadienesulfonyl]prop-2-yl 2-bromoacrylate (14g).}

The title compound $\mathbf{1 4 g}(28 \%)$ was obtained by procedure 1.9 from $\beta$-hydroxy sulfone 4 and 2bromoacrylic acid (13) after column chromatography (EtOAc/Hexane, 1/2, $\mathrm{R}_{\mathrm{f}}=0.20$ ).

${ }^{1} \mathrm{H}$ NMR $\left(\mathrm{CDCl}_{3}\right) \delta 1.47\left(\mathrm{~d}, \mathrm{~J}=6.4 \mathrm{~Hz}, 3 \mathrm{H}, \mathrm{CH}_{3}\right), 3.21\left(\mathrm{dd}, \mathrm{J}=14.9,4.0 \mathrm{~Hz}, 1 \mathrm{H}, \mathrm{H}^{2 \alpha}\right), 3.47(\mathrm{dd}$, $\left.\mathrm{J}=14.9,8.0 \mathrm{~Hz}, 1 \mathrm{H}, \mathrm{H}^{2 \beta}\right), 5.43\left(\mathrm{ddq}, \mathrm{J}=8.0,4.0,6.5 \mathrm{~Hz}, 1 \mathrm{H}, \mathrm{H}^{1}\right), 5.66\left(\mathrm{br} \mathrm{d}, \mathrm{J}=9.9 \mathrm{~Hz}, 1 \mathrm{H}, \mathrm{H}^{6 \mathrm{a}}\right)$, 5.75 (br d, J=16.8 Hz, 1H, H $\left.{ }^{6 b}\right), 6.30\left(\mathrm{~d}, \mathrm{~J}=1.8 \mathrm{~Hz}, 1 \mathrm{H}, \mathrm{H}^{8 \mathrm{a}}\right), 6.40\left(\mathrm{br} \mathrm{d}, \mathrm{J}=14.9 \mathrm{~Hz}, 1 \mathrm{H}, \mathrm{H}^{3}\right), 6.44$ (dddd, $\left.\mathrm{J}=16.9,10.9,10.0,0.6 \mathrm{~Hz}, 1 \mathrm{H}, \mathrm{H}^{5}\right), 6.98\left(\mathrm{~d}, \mathrm{~J}=1.8 \mathrm{~Hz}, 1 \mathrm{H}, \mathrm{H}^{8 \mathrm{~b}}\right), 7.17(\mathrm{br} \mathrm{dd}, \mathrm{J}=14.9,10.9$ $\left.\mathrm{Hz}, 1 \mathrm{H}, \mathrm{H}^{4}\right) ;{ }^{13} \mathrm{C} \mathrm{NMR}\left(\mathrm{CDCl}_{3}\right) \delta 20.2,59.8,68.2,120.8,128.7,129.4,131.9,132.3,144.9$, 160.8 .

\subsection{2-[(E)-Butadienesulfonyl]-1-phenylethyl 2-bromoacrylate (14h).}

2-Bromoacrylic acid (13) ( $0.780 \mathrm{~g}, 5.17 \mathrm{mmol}, 1.88$ equiv) was dissolved in anhydrous THF (12 $\mathrm{mL})$ and cooled to $0{ }^{\circ} \mathrm{C}$. Triethylamine $(1.52 \mathrm{~mL}, 1.09 \mathrm{~g}, 10.8 \mathrm{mmol}, 3.92$ equiv) was added dropwise with stirring. The reaction mixture was stirred at $0{ }^{\circ} \mathrm{C}$ for $10 \mathrm{~min}$, then $2,4,6-$ trichlorobenzoyl chloride $(0.78 \mathrm{~mL}, 1.22 \mathrm{~g}, 4.99 \mathrm{mmol}, 1.81$ equiv $)$ was added. The reaction mixture was stirred at $0{ }^{\circ} \mathrm{C}$ for $1 \mathrm{~h} 20 \mathrm{~min}$, when it became dark brown. Solid $\mathrm{LiCl}(0.293 \mathrm{~g}, 6.91$ mmol, 2.51 equiv) was added, followed by a solution of $\beta$-hydroxy sulfone 9 ( $0.656 \mathrm{~g}, 2.75$ mmol, 1 equiv) in anhydrous THF $(12 \mathrm{~mL})$. The reaction mixture was stirred for $15 \mathrm{~h}$ at $\mathrm{rt}$ and was then diluted with ether $(75 \mathrm{~mL})$ and filtered. The filtrate was diluted with EtOAc $(50 \mathrm{~mL})$ and refiltered. The filtrate was concentrated in vacuo and immediately subjected to column chromatography (EtOAc/Hexane, $1 / 3)$ to give the title compound $\mathbf{1 4 h}$ as a colorless oil $(0.484 \mathrm{~g}$, $1.30 \mathrm{mmol}, 47 \%)$ and recovered $\beta$-hydroxy sulfone $9(0.132 \mathrm{~g}, 0.55 \mathrm{mmol}, 20 \%)$.

14h: ${ }^{1} \mathrm{H}$ NMR $\left(\mathrm{CDCl}_{3}\right) \delta 3.39\left(\mathrm{dd}, \mathrm{J}=15.1,3.1 \mathrm{~Hz}, 1 \mathrm{H}, \mathrm{H}^{2 \alpha}\right), 3.80\left(\mathrm{dd}, \mathrm{J}=15.1,9.8 \mathrm{~Hz}, 1 \mathrm{H}, \mathrm{H}^{2 \beta}\right)$, $5.66\left(\right.$ br d, J=10.0 Hz, 1H, H $\left.{ }^{6 a}\right), 5.74\left(\right.$ br d, J=16.9 Hz, $\left.1 \mathrm{H}, \mathrm{H}^{6 \mathrm{~b}}\right), 6.29\left(\mathrm{~d}, \mathrm{~J}=1.8 \mathrm{~Hz}, 1 \mathrm{H}, \mathrm{H}^{8 \mathrm{a}}\right.$ ), $6.30\left(\mathrm{dd}, \mathrm{J}=9.9,3.1 \mathrm{~Hz}, 1 \mathrm{H}, \mathrm{H}^{1}\right), 6.34\left(\mathrm{br} \mathrm{d}, \mathrm{J}=14.9 \mathrm{~Hz}, 1 \mathrm{H}, \mathrm{H}^{3}\right), 6.40($ app dt, J=16.9, $10.4 \mathrm{~Hz}$, $\left.1 \mathrm{H}, \mathrm{H}^{5}\right), 7.02\left(\mathrm{~d}, \mathrm{~J}=1.8 \mathrm{~Hz}, 1 \mathrm{H}, \mathrm{H}^{8 \mathrm{~b}}\right), 7.16\left(\mathrm{dd}, \mathrm{J}=14.9,10.9 \mathrm{~Hz}, 1 \mathrm{H}, \mathrm{H}^{4}\right), 7.27-7.45(\mathrm{~m}, 5 \mathrm{H}, \mathrm{Ph})$; ${ }^{13} \mathrm{C} \mathrm{NMR}\left(\mathrm{CDCl}_{3}\right) \delta 60.4,72.9,120.4,126.7,128.8,129.3,129.4,129.5,132.28,132.31,137.0$, $144.9,160.4$.

\subsection{2-[(E)-Butadienesulfonyl]ethyl acrylate (14i).}

The title compound $14 \mathbf{i}(85 \%)$ was obtained by procedure 1.9 from $\beta$-hydroxy sulfone 3 and acrylic acid (12) after column chromatography (EtOAc/Hexane, 1/2.5).

${ }^{1} \mathrm{H} \mathrm{NMR}\left(\mathrm{CDCl}_{3}\right) \delta 3.41$ (app t, J=6.0 Hz, 2H, H$\left.{ }^{2}\right), 4.56\left(\right.$ app t, J=6.0 Hz, 2H, $\left.\mathrm{H}^{1}\right), 5.68$ (br d, $\left.\mathrm{J}=9.9 \mathrm{~Hz}, 1 \mathrm{H}, \mathrm{H}^{6 \mathrm{a}}\right), 5.77\left(\mathrm{br} \mathrm{d}, \mathrm{J}=16.9 \mathrm{~Hz}, 1 \mathrm{H}, \mathrm{H}^{6 \mathrm{~b}}\right), 5.89\left(\mathrm{dd}, \mathrm{J}=10.4,1.4 \mathrm{~Hz}, 1 \mathrm{H}, \mathrm{H}^{8 \mathrm{a}}\right), 6.09$ (dd, $\left.\mathrm{J}=17.3,10.4 \mathrm{~Hz}, 1 \mathrm{H}, \mathrm{H}^{7}\right), 6.41\left(\mathrm{brd}, \mathrm{J}=14.9 \mathrm{~Hz}, 1 \mathrm{H}, \mathrm{H}^{3}\right), 6.43\left(\mathrm{dd}, \mathrm{J}=17.3,1.4 \mathrm{~Hz}, 1 \mathrm{H}, \mathrm{H}^{8 \mathrm{~b}}\right.$ ), $6.45\left(\operatorname{app~dt}, \mathrm{J}=16.9,10.5 \mathrm{~Hz}, 1 \mathrm{H}, \mathrm{H}^{5}\right), 7.20\left(\mathrm{dd}, \mathrm{J}=14.9,10.9 \mathrm{~Hz}, 1 \mathrm{H}, \mathrm{H}^{4}\right)$. 


\subsection{General procedure for intramolecular Diels-Alder cycloaddition of $\beta$ - acyloxy sulfones 14a-h.}

A solution of $\beta$-acyloxy sulfone 14a-h and 2,5-di-tert-butyl-4-methylphenol (5-7 mol \%) in toluene or xylenes (bp 136-140 ${ }^{\circ} \mathrm{C}$ ) was heated in a $150 \mathrm{~mL}$ glass pressure vessel (time and temperature indicated in Table 2). After cooling to rt, the reaction mixture was filtered and the filtered solid was washed with toluene. The combined filtrates were concentrated in vacuo and the residue was purified by either crystallization or column chromatography.

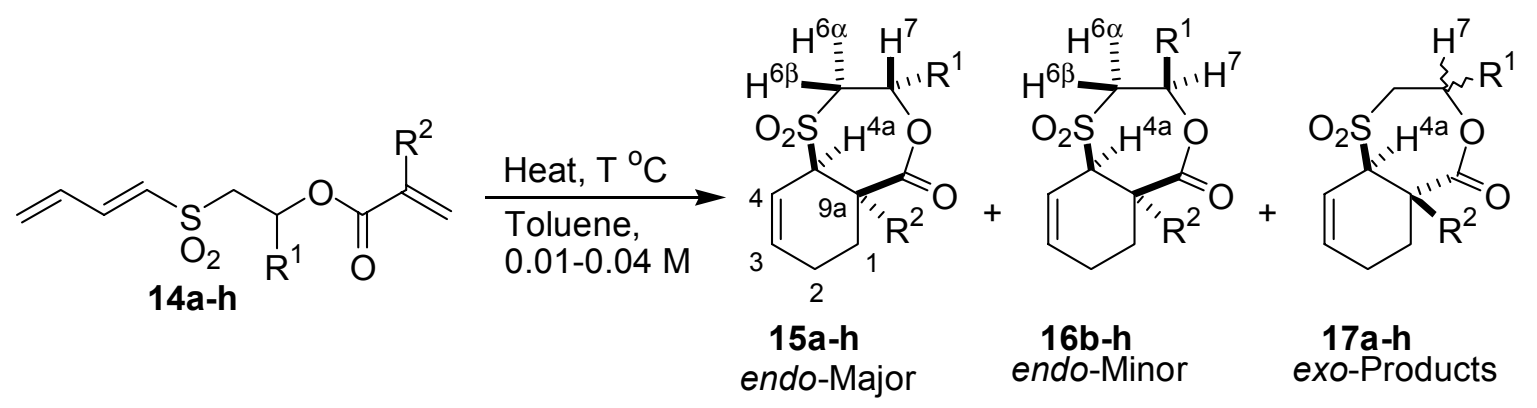

a $R^{1}=H, R^{2}=M e ; \quad$ b $R^{1}=M e, R^{2}=M e ; \quad c R^{1}=P h, R^{2}=M e ; \quad d R^{1}=t-B u, R^{2}=M e ;$

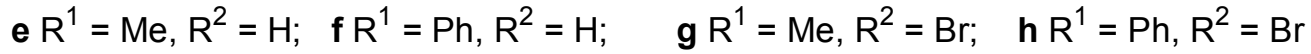

The presence and amounts of exo-products $\mathbf{1 7 b}, \mathbf{c}, \mathbf{g}, \mathbf{h}$ were determined by the presence and integration of $\mathrm{H}^{4 \mathrm{a}}$ in the ${ }^{1} \mathrm{H}$ NMR spectrum of crude reaction mixtures (Table $\mathrm{S} 2$ ).

Table S2. Chemical shifts of $\mathrm{H}^{4 \mathrm{a}}$ proton in cycloadducts 15-17

\begin{tabular}{|l|l|l|l|l|l|l|l|l|}
\hline \multirow{2}{*}{ Compound } & \multicolumn{9}{|c|}{$\delta \mathrm{H}^{4 \mathrm{a}}, \mathrm{ppm}$} \\
\cline { 2 - 9 } & $\mathbf{a}$ & $\mathbf{b}$ & $\mathbf{c}$ & $\mathbf{d}$ & $\mathbf{e}$ & $\mathbf{f}$ & $\mathbf{g}$ & $\mathbf{h}$ \\
\hline $\mathbf{1 5}$ & 3.67 & 3.70 & 3.85 & 3.71 & 4.12 & 4.26 & 4.32 & 4.47 \\
\hline $\mathbf{1 6}$ & N/A & 3.56 & 3.71 & 3.63 & 3.89 & 3.98 & 4.44 & 4.74 \\
\hline $\mathbf{1 7}$ & 3.92 & 3.94 & 4.15 & 4.26 & --- & --- & 4.51 & 4.80 \\
\hline
\end{tabular}

As above, the protons $\mathrm{H}^{6 \alpha}, \mathrm{H}^{6 \beta}$ and $\mathrm{H}^{7}$ in the cycloadducts compose AMX or ABX coupling systems. For those exhibiting $A B X$ systems, apparent values of $J_{A X}$ and $J_{B X}$, presented in the text below, are dependent on the spectrometer frequency. The $\Delta v / J_{A B}$ values are given in Table S3.

Table S3. $\Delta v / J_{\mathrm{AB}}$ values for cycloadducts 15-17 and decomposition product $\mathbf{1 8 g}$

\begin{tabular}{|l|l|l|l|l|l|l|l|l|l|l|l|l|l|l|l|}
\hline Comp. & $\mathbf{1 5 b}$ & $\mathbf{1 6 b}$ & $\mathbf{1 5 c}$ & $\mathbf{1 6 c}$ & $\mathbf{1 5 d}$ & $\mathbf{1 6 d}$ & $\mathbf{1 7 d}$ & $\mathbf{1 5 e}$ & $\mathbf{1 6 e}$ & $\mathbf{1 5 f}$ & $\mathbf{1 6 f}$ & $\mathbf{1 5 g}$ & $\mathbf{1 6 g}$ & $\mathbf{1 8 g}$ & $\mathbf{1 5 h}$ \\
\hline$\Delta v / \mathrm{J}_{\mathrm{AB}}$ & 3.08 & 7.0 & 5.3 & 17 & 7.4 & 14.7 & 2.1 & 2.9 & 5.0 & 5.8 & 11 & 2.7 & 3.1 & 0.85 & 4.6 \\
\hline
\end{tabular}

\subsection{1. (4aSR,9aSR)-9a-Methyl-5,5-dioxo-1,2,4a,9a-tetrahydro-8-oxa-5 $\lambda^{6}$-thiabenzo- cycloheptan-9-one (15a).}

$\beta$-Acyloxy sulfone 14a $(0.85 \mathrm{~g}, 3.69 \mathrm{mmol})$ was heated in toluene $(90 \mathrm{~mL}, 0.041 \mathrm{M})$ at $130{ }^{\circ} \mathrm{C}$ for $29.5 \mathrm{~h}$. Column chromatography (EtOAc/Hexanes, 1/1), gave a 100/33 mixture of cycloadducts $15 \mathbf{a}$ and $17 \mathbf{a}(0.36 \mathrm{~g}, 1.56 \mathrm{mmol}, 42 \%)$. A clean sample of the title compound $15 \mathbf{a}$ was obtained as a white crystalline solid by crystallization of the mixture from EtOH, mp 139$141^{\circ} \mathrm{C}$.

${ }^{1} \mathrm{H}$ NMR $\left(\mathrm{CDCl}_{3}\right) \delta 1.34\left(\mathrm{~s}, 3 \mathrm{H}, \mathrm{CH}_{3}\right), 1.79-1.89\left(\mathrm{~m}, 1 \mathrm{H}, \mathrm{H}^{1} / \mathrm{H}^{2}\right), 2.04-2.43\left(\mathrm{~m}, 3 \mathrm{H}, \mathrm{H}^{1} / \mathrm{H}^{2}\right), 3.29$ (ddd, J=14.5, 3.2, 2.4 Hz, $\left.1 \mathrm{H}, \mathrm{H}^{6}\right), 3.55\left(\mathrm{ddd}, \mathrm{J}=14.5,11.0,5.5 \mathrm{~Hz}, 1 \mathrm{H}, \mathrm{H}^{6}\right), 3.67\left(\right.$ br s, $1 \mathrm{H}, \mathrm{H}^{4 \mathrm{a}}$ ), 4.47 (ddd, J=12.3, 5.5, $\left.2.3 \mathrm{~Hz}, 1 \mathrm{H}, \mathrm{H}^{7}\right), 4.64\left(\mathrm{ddd}, \mathrm{J}=12.3,11.0,3.4 \mathrm{~Hz}, 1 \mathrm{H}, \mathrm{H}^{7}\right), 5.83-5.91(\mathrm{~m}$, $\left.1 \mathrm{H}, \mathrm{H}^{4}\right), 6.24-6.32\left(\mathrm{~m}, 1 \mathrm{H}, \mathrm{H}^{3}\right) ;{ }^{13} \mathrm{C} \mathrm{NMR}\left(\mathrm{CDCl}_{3}\right.$, one signal masked) $\delta 22.2,26.1,43.9,57.2$, $64.1,67.3,114.7,135.9,179.2$; X-ray crystal structure reported in ref. 21a. 
(4aSR,9aRS)-9a-Methyl-5,5-dioxo-1,2,4a,9a-tetrahydro-8-oxa-5 $\lambda^{6}$-thiabenzocycloheptan-9one (17a).

The mother liquor remaining after the crystallization of compound 15a (Experiment 1.18.1) was concentrated in vacuo to obtain a 100/75 mixture of $\mathbf{1 5 a}$ and 17a as a white solid. Spectral data of the title compound 17a was assigned from this mixture:

${ }^{1} \mathrm{H} \mathrm{NMR}\left(\mathrm{CDCl}_{3}\right) \delta 1.54\left(\mathrm{~s}, 3 \mathrm{H}, \mathrm{CH}_{3}\right), 1.80-2.38\left(\mathrm{~m}, 4 \mathrm{H}, \mathrm{H}^{1} / \mathrm{H}^{2}\right), 3.41(\mathrm{br} \mathrm{dd}, \mathrm{J}=15.6,5.1 \mathrm{~Hz}$, $\left.1 \mathrm{H}, \mathrm{H}^{6}\right), 3.55$ (ddd, J=15.7, 10.5, $\left.2.4 \mathrm{~Hz}, 1 \mathrm{H}, \mathrm{H}^{6}\right), 3.92\left(\right.$ br s, $\left.1 \mathrm{H}, \mathrm{H}^{4 \mathrm{a}}\right), 4.59$ (ddd, J=14.4, 5.4, 2.3 $\left.\mathrm{Hz}, 1 \mathrm{H}, \mathrm{H}^{7}\right), 5.03\left(\mathrm{br} \mathrm{dd}, \mathrm{J}=14.5,10.6 \mathrm{~Hz}, 1 \mathrm{H}, \mathrm{H}^{7}\right), 5.99-6.07\left(\mathrm{~m}, 1 \mathrm{H}, \mathrm{H}^{4}\right), 6.16-6.23(\mathrm{~m}, 1 \mathrm{H}$, $\left.\mathrm{H}^{3}\right) ;{ }^{13} \mathrm{C} \mathrm{NMR}\left(\mathrm{CDCl}_{3}\right) \delta 15.2,21.9,33.9,43.1,54.8,61.5,64.3,113.8,133.4,174.9$.

\subsection{2. (4aSR,7RS,9aSR)-7,9a-Dimethyl-5,5-dioxo-1,2,4a,9a-tetrahydro-8-oxa-5 $\lambda^{6}$ - thiabenzocycloheptan-9-one (15b).}

$\beta$-Acyloxy sulfone $14 \mathrm{~b}(1.25 \mathrm{~g}, 5.12 \mathrm{mmol})$ was heated in xylenes $(500 \mathrm{~mL}, 0.010 \mathrm{M})$ at $140{ }^{\circ} \mathrm{C}$ for $41 \mathrm{~h}$. Column chromatography (EtOAc/Hexanes, 1/1.5), gave a 100/25 mixture of cycloadducts $15 \mathrm{~b}$ and $\mathbf{1 6 b}(0.70 \mathrm{~g}, 2.87 \mathrm{mmol}, 56 \%)$ as a white solid. Crystallization of the mixture from EtOAc/Hexanes $(1 / 2,12 \mathrm{~mL})$ gave the title compound $\mathbf{1 5 b}$ as a white crystalline solid (0.45 g, $1.84 \mathrm{mmol}, 36 \%)$, mp $149^{\circ} \mathrm{C}$.

${ }^{1} \mathrm{H}$ NMR $\left(400 \mathrm{MHz}, \mathrm{CDCl}_{3}\right) \delta 1.31\left(\mathrm{~s}, 3 \mathrm{H}, \mathrm{CH}_{3}\right), 1.52\left(\mathrm{~d}, \mathrm{~J}=6.4 \mathrm{~Hz}, 3 \mathrm{H}, \mathrm{CH}_{3}\right), 1.82-1.90(\mathrm{~m}, 1 \mathrm{H}$, $\left.\mathrm{H}^{1} / \mathrm{H}^{2}\right), 2.05-3.36\left(\mathrm{~m}, 3 \mathrm{H}, \mathrm{H}^{1} / \mathrm{H}^{2}\right), 3.25\left(\mathrm{dd}, \mathrm{J}=14.3,2.9 \mathrm{~Hz}, 1 \mathrm{H}, \mathrm{H}^{6 \beta}\right), 3.36(\mathrm{dd}, \mathrm{J}=14.3,11.1 \mathrm{~Hz}$, $\left.1 \mathrm{H}, \mathrm{H}^{6 \alpha}\right), 3.70\left(\right.$ br s, $\left.1 \mathrm{H}, \mathrm{H}^{4 \mathrm{a}}\right), 4.99\left(\mathrm{ddq}, \mathrm{J}=11.1,2.9,6.4 \mathrm{~Hz}, 1 \mathrm{H}, \mathrm{H}^{7}\right), 5.83-5.89\left(\mathrm{~m}, 1 \mathrm{H}, \mathrm{H}^{4}\right)$, 6.24-6.31 (m, $\left.1 \mathrm{H}, \mathrm{H}^{3}\right) ;{ }^{13} \mathrm{C} \mathrm{NMR}\left(\mathrm{CDCl}_{3}\right) \delta 20.2,21.7,22.1,25.1,43.9,64.5,66.5,73.1,114.3$, $135.9,179.0, \mathrm{X}$-ray crystal structure reported in ref. $21 \mathrm{~b}$.

\section{(4aSR,7SR,9aSR)-7,9a-Dimethyl-5,5-dioxo-1,2,4a,9a-tetrahydro-8-oxa-5 $\lambda^{6}$ - thiabenzocycloheptan-9-one (16b).}

White solid, spectral data assigned from the mixture of $\mathbf{1 6 b} / \mathbf{1 7} \mathbf{b}$ obtained as a second fraction after column chromatography by procedure 1.18.2.

${ }^{1} \mathrm{H} \mathrm{NMR}\left(\mathrm{CDCl}_{3}\right) \delta 1.49\left(\mathrm{~s}, 3 \mathrm{H}, \mathrm{CH}_{3}\right), 1.48\left(\mathrm{~d}^{\approx}, 3 \mathrm{H}, \mathrm{CH}_{3}\right), 1.62-1.76\left(\mathrm{~m}, 1 \mathrm{H}, \mathrm{H}^{1} / \mathrm{H}^{2}\right), 2.01-2.38$ $\left(\mathrm{m}, 3 \mathrm{H}, \mathrm{H}^{1} / \mathrm{H}^{2}\right), 3.09$ (ddd, $\left.\mathrm{J}=14.5,3.4,1.1 \mathrm{~Hz}, 1 \mathrm{H}, \mathrm{H}^{6 \alpha}\right), 3.43\left(\mathrm{dd}, \mathrm{J}=14.5,9.0 \mathrm{~Hz}, 1 \mathrm{H}, \mathrm{H}^{6 \beta}\right), 3.56$ (br s, $\left.1 \mathrm{H}, \mathrm{H}^{4 \mathrm{a}}\right)$, $\left(\mathrm{H}^{7}\right.$ is hidden by $\mathrm{H}^{7}$ of $\left.\mathbf{1 5 b}\right), 5.80-5.93\left(\mathrm{~m}, 1 \mathrm{H}, \mathrm{H}^{4}\right), 6.23-6.32\left(\mathrm{~m}, 1 \mathrm{H}, \mathrm{H}^{3}\right)$; ${ }^{13} \mathrm{C}$ $\operatorname{NMR}\left(\mathrm{CDCl}_{3}\right) \delta 20.1,22.4,25.3,31.7,45.0,58.5,70.1,72.1,118.0,135.3(\mathrm{C}=\mathrm{O}$ was not observed).

\subsection{3. (4aSR,7SR,9aSR)-7-Phenyl-9a-methyl-5,5-dioxo-1,2,4a,9a-tetrahydro-8-oxa-5 $\lambda^{6}$ - thiabenzocycloheptan-9-one (15c).}

$\beta$-Acyloxy sulfone 14c $(0.84 \mathrm{~g}, 2.74 \mathrm{mmol})$ was heated in toluene $(250 \mathrm{~mL}, 0.011 \mathrm{M})$. After $15 \mathrm{~h}$ at $118{ }^{\circ} \mathrm{C}$, conversion to cycloadduct was less than $25 \%\left({ }^{1} \mathrm{H}\right.$ NMR analysis). Heating was continued for an additional $24 \mathrm{~h}$ at $138{ }^{\circ} \mathrm{C}$ - conversion to cycloadduct was $87 \%\left({ }^{1} \mathrm{H}\right.$ NMR analysis). Removal of the solvent afforded a 100/25/10/20 mixture of cycloadducts 15c, 16c, 17c and $\beta$-acyloxy sulfone 14c. Crystallization of the crude reaction mixture from EtOAc/Hexanes $(1 / 3)$ gave the clean title compound $15 \mathrm{c}$ as a white solid, mp $208-209^{\circ} \mathrm{C}(0.41 \mathrm{~g}, 1.34 \mathrm{mmol}$, $49 \%$ ). Identical results were obtained if the reaction was performed for $28 \mathrm{~h}$ straight at $138{ }^{\circ} \mathrm{C}$ (Table 2, entry 4).

If the reaction was performed for $50 \mathrm{~h}$ at $145^{\circ} \mathrm{C}(0.017 \mathrm{M}$ in toluene) (Table 2, entry 5$)$, the conversion of $\mathbf{1 4 c}$ to cycloadducts was complete ( ${ }^{1} \mathrm{H}$ NMR analysis), however, the yield of $\mathbf{1 5 c}$ remained $49 \%$, while the crystallization was more difficult.

${ }^{1} \mathrm{H}$ NMR $\left(400 \mathrm{MHz}, \mathrm{CDCl}_{3}\right) \delta 1.39\left(\mathrm{~s}, 3 \mathrm{H}, \mathrm{CH}_{3}\right), 1.89-1.97\left(\mathrm{~m}, 1 \mathrm{H}, \mathrm{H}^{1}\right), 2.07-2.20\left(\mathrm{~m}, 1 \mathrm{H}, \mathrm{H}^{1}\right)$, 2.24-2.40 (m, 2H, $\left.\mathrm{H}^{2}\right), 3.52\left(\mathrm{br} \mathrm{dd}, \mathrm{J}=14.3,2.9 \mathrm{~Hz}, 1 \mathrm{H}, \mathrm{H}^{6 \beta}\right), 3.71(\mathrm{dd}, \mathrm{J}=14.3,11.6 \mathrm{~Hz}, 1 \mathrm{H}$, $\left.\mathrm{H}^{6 \alpha}\right), 3.85\left(\right.$ br s, $\left.1 \mathrm{H}, \mathrm{H}^{4 \mathrm{a}}\right), 5.87\left(\mathrm{dd}, \mathrm{J}=11.5,2.8 \mathrm{~Hz}, 1 \mathrm{H}, \mathrm{H}^{7}\right), 5.88-5.93\left(\mathrm{~m}, 1 \mathrm{H}, \mathrm{H}^{4}\right), 6.28-6.34$

\footnotetext{
$\approx$ The value of the coupling constant was not calculated because of overlapping signals.
} 
$\left(\mathrm{m}, 1 \mathrm{H}, \mathrm{H}^{3}\right), 7.36-7.46(\mathrm{~m}, 5 \mathrm{H}, \mathrm{Ph}) ;{ }^{13} \mathrm{C} \mathrm{NMR}\left(\mathrm{CDCl}_{3}\right.$, one signal masked) $\delta 21.8,22.1,25.3$, 44.1, 64.1, 67.0, 77.2, 114.2, 126.0, 129.2, 129.3, 136.1, 136.2, 178.4.

NOE (400 MHz): irradiation of the $\mathrm{CH}_{3}$ group resulted in enhancement of the two $\mathrm{H}^{1}$ protons and strong enhancement of $\mathrm{H}^{4 a}$; irradiation of $\mathrm{H}^{4 a}$ resulted in strong enhancement of $\mathrm{H}^{4}$ and a weak negative NOE of $\mathrm{H}^{3}$; irradiation of $\mathrm{H}^{6 \alpha}$ resulted in enhancement of $\mathrm{H}^{4 a}$ and $\mathrm{H}^{6 \beta}$; irradiation of $\mathrm{H}^{6 \beta}$ resulted in strong enhancement of $\mathrm{H}^{7}$ and $\mathrm{H}^{6 \alpha}$ and a weak negative NOE of $\mathrm{H}^{4 \mathrm{a}}$.

(4aSR,7RS,9aSR)-9a-Methyl-7-phenyl-5,5-dioxo-1,2,4a,9a-tetrahydro-8-oxa-5 $\lambda^{6}$ thiabenzocycloheptan-9-one (16c).

Spectral data was partially assigned from the white solid mixture of $\mathbf{1 5} \mathbf{c} / \mathbf{1 6} \mathbf{c}$ obtained from further crystallization of the mother liquer in product 1.18.3: ${ }^{1} \mathrm{H} \mathrm{NMR}\left(400 \mathrm{MHz}, \mathrm{CDCl}_{3}\right)$ (partial listing) $\delta 1.60\left(\mathrm{~s}, 3 \mathrm{H}, \mathrm{CH}_{3}\right), 1.78\left(\mathrm{ddd}, \mathrm{J}=14.0,11.9,5.2 \mathrm{~Hz}, 1 \mathrm{H}, \mathrm{H}^{1} / \mathrm{H}^{2}\right), 2.06-2.47(\mathrm{~m}$, $\left.3 \mathrm{H}, \mathrm{H}^{1} / \mathrm{H}^{2}\right), 3.19$ (ddd, $\left.\mathrm{J}=14.4,2.9,1.4 \mathrm{~Hz}, 1 \mathrm{H}, \mathrm{H}^{6 \alpha}\right), 3.71$ (br s, $\left.1 \mathrm{H}, \mathrm{H}^{4 \mathrm{a}}\right), 3.79$ (dd, J=14.5, 11.1 $\left.\mathrm{Hz}, 1 \mathrm{H}, \mathrm{H}^{6 \beta}\right), 5.79\left(\mathrm{dd}, \mathrm{J}=11.0,2.9,1 \mathrm{H}, \mathrm{H}^{7}\right), 5.92-5.98\left(\mathrm{~m}, 1 \mathrm{H}, \mathrm{H}^{4}\right), 6.32-6.39\left(\mathrm{~m}, 1 \mathrm{H}, \mathrm{H}^{3}\right), 7.28-$ $7.33(\mathrm{~m}, 2 \mathrm{H}, \mathrm{Ph}) ;{ }^{13} \mathrm{C}$ NMR $\left(\mathrm{CDCl}_{3}\right.$, one signal around $77 \mathrm{ppm}$ and one signal of Ph are masked) $\delta 22.9,26.8,33.5,45.2,58.6,71.5,119.5,129.2,129.3,135.0,136.4,177.3$.

\subsection{4. (4aSR,7SR,9aSR)-7-t-Butyl-9a-methyl-5,5-dioxo-1,2,4a,9a-tetrahydro-8-oxa-5 $\lambda^{6}$ - thiabenzocycloheptan-9-one (15d).}

$\beta$-Acyloxy sulfone 14d $(0.478 \mathrm{~g}, 1.67 \mathrm{mmol})$ was heated in toluene $(150 \mathrm{~mL}, 0.011 \mathrm{M})$ at $138{ }^{\circ} \mathrm{C}$ for $47 \mathrm{~h}$. Crystallization of the crude reaction mixture from EtOAc/Hexanes $(1 / 3,40 \mathrm{~mL})$ gave the title compound $15 \mathrm{~d}$ as a white crystalline solid $(0.261 \mathrm{~g}, 0.91 \mathrm{mmol}, 55 \%), \mathrm{mp} 216-217{ }^{\circ} \mathrm{C}$, turns light yellow on melting. Recrystallization of the concentrated mother liquor from EtOAc/Hexanes $(1 / 3,7 \mathrm{~mL})$ gave a second crop of clean $15 d(0.052 \mathrm{~g}, 0.18 \mathrm{mmol}, 11 \%), \mathrm{mp}$ $216-217^{\circ} \mathrm{C}$, turns light yellow. Total yield of the title compound $15 \mathrm{~d}$ was $0.313 \mathrm{~g}, 1.09 \mathrm{mmol}$, $65 \%$.

${ }^{1} \mathrm{H}$ NMR $\left(400 \mathrm{MHz}, \mathrm{CDCl}_{3}\right) \delta 1.04(\mathrm{~s}, 9 \mathrm{H}, t-\mathrm{Bu}), 1.32\left(\mathrm{~s}, 3 \mathrm{H}, \mathrm{CH}_{3}\right), 1.84-1.91\left(\mathrm{~m}, 1 \mathrm{H}, \mathrm{H}^{1}\right), 2.05-$ $2.36\left(\mathrm{~m}, 3 \mathrm{H}, \mathrm{H}^{1} / \mathrm{H}^{2}\right), 3.21\left(\mathrm{dd}, \mathrm{J}=14.1,2.6 \mathrm{~Hz}, 1 \mathrm{H}, \mathrm{H}^{6 \beta}\right), 3.47\left(\mathrm{dd}, \mathrm{J}=14.1,11.8 \mathrm{~Hz}, 1 \mathrm{H}, \mathrm{H}^{6 \alpha}\right)$, $3.71\left(\right.$ br s, $\left.1 \mathrm{H}, \mathrm{H}^{4 \mathrm{a}}\right), 4.50\left(\mathrm{dd}, \mathrm{J}=11.8,2.6 \mathrm{~Hz}, 1 \mathrm{H}, \mathrm{H}^{7}\right), 5.84-5.90\left(\mathrm{~m}, 1 \mathrm{H}, \mathrm{H}^{4}\right), 6.24-6.30(\mathrm{~m}, 1 \mathrm{H}$, $\left.\mathrm{H}^{3}\right) ;{ }^{13} \mathrm{C} \mathrm{NMR}\left(\mathrm{CDCl}_{3}\right) \delta 22.0,22.1,25.2,25.8,34.2,44.1,59.6,67.0,83.6,114.4,135.9,179.0$. NOE (400 MHz): irradiation of Me resulted in strong enhancement of $\mathrm{H}^{4 \mathrm{a}}$ and enhancement of $\mathrm{H}^{1}$ at 1.84-1.91 ppm; irradiation of $\mathrm{H}^{4 a}$ resulted in strong enhancement of $\mathrm{H}^{4}$ and weak enhancement of $\mathrm{H}^{6 \alpha}$; irradiation of $\mathrm{H}^{6 \alpha}$ resulted in enhancement of $\mathrm{H}^{4 a}$ and $\mathrm{H}^{6 \beta}$; irradiation of $\mathrm{H}^{6 \beta}$ resulted in enhancement of $\mathrm{H}^{7}$ and $\mathrm{H}^{6 \alpha}$; irradiation of $\mathrm{H}^{7}$ resulted in weak enhancement of $\mathrm{H}^{6 \beta}$.

(4aSR,7RS,9aSR)-7-t-Butyl-9a-methyl-5,5-dioxo-1,2,4a,9a-tetrahydro-8-oxa-5 $\lambda^{6}$ thiabenzocycloheptan-9-one (16d).

The residue obtained by concentration of the mother liquor after the second crystallization in procedure 1.18.4 was subjected to column chromatography (dichloromethane). The first fraction gave $0.030 \mathrm{~g}(0.10 \mathrm{mmol}, 6 \%)$ of almost clean product 15d; the second fraction (shaving) gave $0.047 \mathrm{~g}$ of a mixture of endo-Major 15d / endo-Minor 16d / exo-17d in a 30/23/100 ratio. The spectral data of the title compound $\mathbf{1 6 d}$ were assigned from this mixture.

${ }^{1} \mathrm{H}$ NMR $\left(\mathrm{CDCl}_{3}\right) \delta 0.97(\mathrm{~s}, 9 \mathrm{H}, t-\mathrm{Bu}), 1.54\left(\mathrm{~s}, 3 \mathrm{H}, \mathrm{CH}_{3}\right), 1.65-1.85\left(\mathrm{~m}, 1 \mathrm{H}, \mathrm{H}^{1} / \mathrm{H}^{2}\right), 1.85-2.40$ $\left(\mathrm{m}, 3 \mathrm{H}, \mathrm{H}^{1} / \mathrm{H}^{2}\right), 2.89\left(\mathrm{ddd}, \mathrm{J}=14.1,2.4,1.3 \mathrm{~Hz}, 1 \mathrm{H}, \mathrm{H}^{6 \alpha}\right), 3.58\left(\mathrm{dd}, \mathrm{J}=14.1,11.7 \mathrm{~Hz}, 1 \mathrm{H}, \mathrm{H}^{6 \beta}\right)$, 3.63 (br s, $\left.1 \mathrm{H}, \mathrm{H}^{4 \mathrm{a}}\right), 4.43\left(\mathrm{dd}, \mathrm{J}=11.7,2.4 \mathrm{~Hz}, 1 \mathrm{H}, \mathrm{H}^{7}\right), 5.83-5.93\left(\mathrm{~m}, 1 \mathrm{H}, \mathrm{H}^{4}\right), 6.22-6.32(\mathrm{~m}, 1 \mathrm{H}$, $\left.\mathrm{H}^{3}\right) ;{ }^{13} \mathrm{C} \mathrm{NMR}\left(\mathrm{CDCl}_{3}\right) \delta 22.9,25.7,26.9,33.9,36.1,45.2,53.6,71.9,83.7,119.6,134.6,177.9$. 
(4aSR,9aRS)-7-t-Butyl-9a-methyl-5,5-dioxo-1,2,4a,9a-tetrahydro-8-oxa-5 $\lambda^{6}$ thiabenzocycloheptan-9-one (17d).

The spectral data of the title compound 17d were assigned from the mixture of endo-Major 15d / endo-Minor 16d / exo-17d (30/23/100 ratio) obtained in the procedure described above for $\mathbf{1 6 d}$. ${ }^{1} \mathrm{H}$ NMR $\left(\mathrm{CDCl}_{3}\right) \delta 1.01(\mathrm{~s}, 9 \mathrm{H}, t-\mathrm{Bu}), 1.48\left(\mathrm{~s}, 3 \mathrm{H}, \mathrm{CH}_{3}\right), 1.80-2.35\left(\mathrm{~m}, 4 \mathrm{H}, \mathrm{H}^{1} / \mathrm{H}^{2}\right), 3.38(\mathrm{dd}$, $\left.\mathrm{J}=15.6,8.1 \mathrm{~Hz}, 1 \mathrm{H}, \mathrm{H}^{6}\right), 3.49\left(\mathrm{dd}, \mathrm{J}=15.6,3.3 \mathrm{~Hz}, 1 \mathrm{H}, \mathrm{H}^{6}\right), 4.26\left(\mathrm{br} \mathrm{s}, 1 \mathrm{H}, \mathrm{H}^{4 \mathrm{a}}\right), 4.56(\mathrm{dd}, \mathrm{J}=8.1$, $\left.3.3 \mathrm{~Hz}, 1 \mathrm{H}, \mathrm{H}^{7}\right), 5.89-5.96\left(\mathrm{~m}, 1 \mathrm{H}, \mathrm{H}^{4}\right), 6.08-6.17\left(\mathrm{~m}, 1 \mathrm{H}, \mathrm{H}^{3}\right) ;{ }^{13} \mathrm{C} \mathrm{NMR}\left(\mathrm{CDCl}_{3}\right) \delta 16.6,22.2$, 25.4, 33.5, 44.9, 55.7, 63.0, 81.8, 114.4, 132.4, 177.6.

\subsection{5. (4aSR,7RS,9aSR)-7-Methyl-5,5-dioxo-1,2,4a,9a-tetrahydro-8-oxa-5 $\lambda^{6}$ - thiabenzocycloheptan-9-one (15e).}

$\beta$-Acyloxy sulfone $14 \mathrm{e}(0.79 \mathrm{~g}, 3.43 \mathrm{mmol})$ was heated in toluene $(150 \mathrm{~mL}, 0.019 \mathrm{M})$ at $130{ }^{\circ} \mathrm{C}$ for $43 \mathrm{~h}$. Column chromatography (EtOAc/Hexane, 1/1) gave the title compound 15e as a white solid (0.43 g, $\left.1.87 \mathrm{mmol}, 54 \%, \mathrm{R}_{\mathrm{f}}=0.34\right), \mathrm{mp} 181-184^{\circ} \mathrm{C}$, and the minor endo-product 16e as a white solid $\left(0.14 \mathrm{~g}, 0.61 \mathrm{mmol}, 18 \%, \mathrm{R}_{\mathrm{f}}=0.21\right), \mathrm{mp} 174-175^{\circ} \mathrm{C}$, as separate fractions. Total yield of cycloadducts was $72 \%$.

15e: ${ }^{1} \mathrm{H}$ NMR $\left(\mathrm{CDCl}_{3}\right) \delta 1.52\left(\mathrm{~d}, \mathrm{~J}=6.4 \mathrm{~Hz}, 3 \mathrm{H}, \mathrm{CH}_{3}\right), 2.02-2.21\left(\mathrm{~m}, 3 \mathrm{H}, \mathrm{H}^{1} / \mathrm{H}^{2}\right), 2.28-2.41(\mathrm{~m}$, $\left.1 \mathrm{H}, \mathrm{H}^{1} / \mathrm{H}^{2}\right), 3.00-3.09\left(\mathrm{~m}, 1 \mathrm{H}, \mathrm{H}^{9 \mathrm{a}}\right), 3.27\left(\mathrm{dd}, \mathrm{J}=14.4,2.8 \mathrm{~Hz}, 1 \mathrm{H}, \mathrm{H}^{6 \beta}\right), 3.41(\mathrm{dd}, \mathrm{J}=14.4,10.8$ $\mathrm{Hz}, 1 \mathrm{H}, \mathrm{H}^{6 \alpha}$ ), 4.12 (br s, $1 \mathrm{H}, \mathrm{H}^{4 \mathrm{a}}$ ), 5.00 (ddq, J=10.8, 2.8, 6.4 Hz, 1H, $\mathrm{H}^{7}$ ), 5.89-5.99 (m, 1H, $\left.\mathrm{H}^{4}\right), 6.28-6.39\left(\mathrm{~m}, 1 \mathrm{H}, \mathrm{H}^{3}\right) ;{ }^{13} \mathrm{C} \mathrm{NMR}\left(\mathrm{CDCl}_{3}\right) \delta 19.9,20.4,24.8,41.9,60.3,64.4,73.2,115.4$, 137.1, 175.9 .

NOE $(500 \mathrm{MHz})$ : irradiation of $\mathrm{H}^{4 \mathrm{a}}$ resulted in strong enhancement of $\mathrm{H}^{4}, \mathrm{H}^{6 \alpha}$ and $\mathrm{H}^{9 \mathrm{a}}$; irradiation of $\mathrm{H}^{7}$ resulted in enhancement of $\mathrm{CH}_{3}$ and $\mathrm{H}^{6 \beta}$.

(4aSR,7SR,9aSR)-7-Methyl-5,5-dioxo-1,2,4a,9a-tetrahydro-8-oxa-5 $\lambda^{6}$ thiabenzocycloheptan-9-one (16e).

The title compound 16e was obtained as a white solid in $18 \%$ yield by procedure 1.18.5. ${ }^{1} \mathrm{H}$ NMR $\left(\mathrm{CDCl}_{3}\right) \delta 1.51\left(\mathrm{~d}, \mathrm{~J}=6.5 \mathrm{~Hz}, 3 \mathrm{H}, \mathrm{CH}_{3}\right), 1.89\left(\mathrm{dddd}, \mathrm{J}=13.7,9.7,7.4,4.1 \mathrm{~Hz}, 1 \mathrm{H}, \mathrm{H}^{1}\right)$, 2.10-2.23 (m, $\left.1 \mathrm{H}, \mathrm{H}^{1} / \mathrm{H}^{2}\right), 2.33-2.43\left(\mathrm{~m}, 1 \mathrm{H}, \mathrm{H}^{1} / \mathrm{H}^{2}\right), 2.49-2.65\left(\mathrm{~m}, 1 \mathrm{H}, \mathrm{H}^{1} / \mathrm{H}^{2}\right), 3.13(\mathrm{ddd}$, $\left.\mathrm{J}=15.1,2.6,1.5 \mathrm{~Hz}, 1 \mathrm{H}, \mathrm{H}^{6 \alpha}\right), 3.38\left(\mathrm{dd}, \mathrm{J}=15.2,9.1 \mathrm{~Hz}, 1 \mathrm{H}, \mathrm{H}^{6 \beta}\right), 3.50($ app q, $\mathrm{J}=4.0 \mathrm{~Hz}, 1 \mathrm{H}$, $\left.\mathrm{H}^{9 \mathrm{a}}\right), 3.89\left(\mathrm{br} \mathrm{s}, 1 \mathrm{H}, \mathrm{H}^{4 \mathrm{a}}\right), 4.95\left(\mathrm{ddq}, \mathrm{J}=9.0,1.4,6.5 \mathrm{~Hz}, 1 \mathrm{H}, \mathrm{H}^{7}\right), 5.86-5.93\left(\mathrm{~m}, 1 \mathrm{H}, \mathrm{H}^{4}\right), 6.06-$ $6.14\left(\mathrm{~m}, 1 \mathrm{H}, \mathrm{H}^{3}\right) ;{ }^{13} \mathrm{C} \mathrm{NMR}\left(\mathrm{CDCl}_{3}\right) \delta 21.4,22.6,25.9,39.2,58.1,63.1,70.0,117.2,135.1$, 171.9.

NOE (500 MHz): irradiation of $\mathrm{H}^{4 \mathrm{a}}$ resulted in strong enhancement of $\mathrm{H}^{4}, \mathrm{H}^{9 \mathrm{a}}$ and $\mathrm{H}^{1}(1.89 \mathrm{ppm})$ and in enhancement of $\mathrm{H}^{7}$; irradiation of $\mathrm{H}^{7}$ resulted in strong enhancement of $\mathrm{CH}_{3}$ and $\mathrm{H}^{9 \mathrm{a}}$, and enhancement of $\mathrm{H}^{6 \alpha}$ and $\mathrm{H}^{4 \mathrm{a}}$.

\subsection{6. (4aSR,7SR,9aSR)-7-Phenyl-5,5-dioxo-1,2,4a,9a-tetrahydro-8-oxa-5 $\lambda^{6}$ - thiabenzocycloheptan-9-one (15f).}

$\beta$-Acyloxy sulfone $14 \mathbf{f}(0.733 \mathrm{~g}, 2.51 \mathrm{mmol})$ was heated in toluene $(250 \mathrm{~mL}, 0.010 \mathrm{M})$ at $127{ }^{\circ} \mathrm{C}$ for $43 \mathrm{~h}$. Column chromatography (dichloromethane) gave the clean title compound $\mathbf{1 5 f}$ as a white solid $\left(0.420 \mathrm{~g}, 1.44 \mathrm{mmol}, 57 \%, \mathrm{R}_{\mathrm{f}}=0.27\right)$, $\mathrm{mp} 164-166{ }^{\circ} \mathrm{C}$. A second fraction contained a ca $1 / 1$ mixture of minor endo-product $\mathbf{1 6 f}\left(\mathrm{R}_{\mathrm{f}}=0.19\right)$ and unreacted $\mathbf{1 4 f}\left(\mathrm{R}_{\mathrm{f}}=0.16\right)$.

15f: ${ }^{1} \mathrm{H}$ NMR (400 MHz, $\left.\mathrm{CDCl}_{3}\right) \delta 2.08-2.27\left(\mathrm{~m}, 3 \mathrm{H}, \mathrm{H}^{1} / \mathrm{H}^{2}\right), 2.32-2.44\left(\mathrm{~m}, 1 \mathrm{H}, \mathrm{H}^{1} / \mathrm{H}^{2}\right), 3.10$ $3.18\left(\mathrm{~m}, 1 \mathrm{H}, \mathrm{H}^{9 \mathrm{a}}\right), 3.52\left(\mathrm{dd}, \mathrm{J}=14.5,2.8 \mathrm{~Hz}, 1 \mathrm{H}, \mathrm{H}^{6 \beta}\right), 3.73\left(\mathrm{dd}, \mathrm{J}=14.4,11.5 \mathrm{~Hz}, 1 \mathrm{H}, \mathrm{H}^{6 \alpha}\right), 4.26$ (br s, $\left.1 \mathrm{H}, \mathrm{H}^{4 \mathrm{a}}\right), 5.87\left(\mathrm{dd}, \mathrm{J}=11.5,2.8 \mathrm{~Hz}, 1 \mathrm{H}, \mathrm{H}^{7}\right), 5.96-6.02\left(\mathrm{~m}, 1 \mathrm{H}, \mathrm{H}^{4}\right), 6.34-6.40\left(\mathrm{~m}, 1 \mathrm{H}, \mathrm{H}^{3}\right)$, 7.35-7.46 (m, 5H, Ph) ${ }^{13} \mathrm{C} \mathrm{NMR}\left(\mathrm{CDCl}_{3}\right) \delta 20.0,24.8,41.9,60.8,64.4,77.6,115.3,126.0$, 129.2, 129.4, 136.2, 137.3, 175.5.

NOE (400 MHz): irradiation of $\mathrm{H}^{9 \mathrm{a}}$ resulted in strong enhancement of $\mathrm{H}^{4 \mathrm{a}}$ and enhancement of $\mathrm{H}^{1}$ around $2.2 \mathrm{ppm}$; irradiation of $\mathrm{H}^{4 a}$ resulted in strong enhancement of $\mathrm{H}^{4}$ and $\mathrm{H}^{9 \mathrm{a}}$, and weak enhancement of $\mathrm{H}^{6 \alpha}$; irradiation of $\mathrm{H}^{6 \alpha}$ resulted in enhancement of $\mathrm{H}^{4 a}$ and $\mathrm{H}^{6 \beta}$; irradiation of 
$\mathrm{H}^{6 \beta}$ resulted in enhancement of $\mathrm{H}^{7}$ and $\mathrm{H}^{6 \alpha}$ and a weak negative NOE of $\mathrm{H}^{4 \mathrm{a}}$; irradiation of $\mathrm{H}^{7}$ resulted in enhancement of $\mathrm{H}^{6 \beta}$ and $\mathrm{Ph}$.

(4aSR,7RS,9aSR)-7-Phenyl-5,5-dioxo-1,2,4a,9a-tetrahydro-8-oxa-5 $\lambda^{6}$ thiabenzocycloheptan-9-one (16f).

The title compound $16 f$ was obtained as a white solid $(0.12 \mathrm{~g}, 0.41 \mathrm{mmol}, 16 \%), \mathrm{mp} 184{ }^{\circ} \mathrm{C}$, by crystallization of the mixture of $\mathbf{1 6 f}$ and $\mathbf{1 4 f}$, obtained by procedure 1.18.6, from EtOAc/Hexane $(1 / 2)$.

${ }^{1} \mathrm{H}$ NMR $\left(400 \mathrm{MHz}, \mathrm{CDCl}_{3}\right) \delta 1.90-2.00\left(\mathrm{~m}, 1 \mathrm{H}, \mathrm{H}^{1}\right), 2.16-2.26\left(\mathrm{~m}, 1 \mathrm{H}, \mathrm{H}^{1} / \mathrm{H}^{2}\right), 2.34-2.42(\mathrm{~m}$, $\left.1 \mathrm{H}, \mathrm{H}^{1} / \mathrm{H}^{2}\right), 2.45-2.58\left(\mathrm{~m}, 1 \mathrm{H}, \mathrm{H}^{1} / \mathrm{H}^{2}\right), 3.31\left(\mathrm{app} \mathrm{dt}, \mathrm{J}=15.0,2.1 \mathrm{~Hz}, 1 \mathrm{H}, \mathrm{H}^{6 \alpha}\right), 3.58-3.64(\mathrm{~m}, 1 \mathrm{H}$, $\left.\mathrm{H}^{9 \mathrm{a}}\right), 3.72\left(\mathrm{dd}, \mathrm{J}=15.0,10.2 \mathrm{~Hz}, 1 \mathrm{H}, \mathrm{H}^{6 \beta}\right), 3.98\left(\right.$ br s, $\left.1 \mathrm{H}, \mathrm{H}^{4 \mathrm{a}}\right), 5.78\left(\mathrm{dd}, \mathrm{J}=10.2,1.6 \mathrm{~Hz}, 1 \mathrm{H}, \mathrm{H}^{7}\right)$, 5.92-5.98 (m, $\left.1 \mathrm{H}, \mathrm{H}^{4}\right), 6.17-6.24\left(\mathrm{~m}, 1 \mathrm{H}, \mathrm{H}^{3}\right) ;{ }^{13} \mathrm{C} \mathrm{NMR}\left(\mathrm{CDCl}_{3}\right) \delta 22.3,25.6,40.0,58.9,63.6$, 75.6, 117.6, 126.0, 129.3, 129.4, 135.1, 136.6, 172.6 .

NOE (400 MHz): irradiation of $\mathrm{H}^{9 \mathrm{a}}$ resulted in strong enhancement of $\mathrm{H}^{4 \mathrm{a}}$ and $\mathrm{H}^{7}$; irradiation of $\mathrm{H}^{4 a}$ resulted in strong enhancement of $\mathrm{H}^{4}$ and $\mathrm{H}^{9 a}$; irradiation of $\mathrm{H}^{6 \alpha}$ resulted in enhancement of $\mathrm{H}^{7}$ and $\mathrm{H}^{6 \beta}$; irradiation of $\mathrm{H}^{7}$ resulted in enhancement of $\mathrm{H}^{9 \mathrm{a}}$.

\subsection{7. (4aSR,7RS,9aRS)-9a-Bromo-7-methyl-5,5-dioxo-1,2,4a,9a-tetrahydro-8-oxa-5 $\lambda^{6}$ - thiabenzocycloheptan-9-one (15g).}

$\beta$-Acyloxy sulfone $14 \mathrm{~g}(0.31 \mathrm{~g}, 1.0 \mathrm{mmol})$ was heated in toluene $(100 \mathrm{~mL}, 0.010 \mathrm{M})$ at $115^{\circ} \mathrm{C}$ for $21 \mathrm{~h}$. Column chromatography (dichloromethane/ether, 40/1) gave the title compound $\mathbf{1 5 g}$ as a yellowish solid, which was clean by ${ }^{1} \mathrm{H}$ and ${ }^{13} \mathrm{C}$ NMR analysis $\left(0.18 \mathrm{~g}, 0.58 \mathrm{mmol}, 58 \%, \mathrm{R}_{\mathrm{f}}=\right.$ $0.37)$. A second fraction contained a ca $100 / 15$ mixture of minor endo-product $16 \mathrm{~g}\left(\mathrm{R}_{\mathrm{f}}=0.22\right)$ and $15 \mathrm{~g}$. The first fraction containing $15 \mathrm{~g}$ was treated with decolorizing charcoal and crystallized from EtOAc/Hexane (1/1) to give the title compound 15g as a white solid $(0.15 \mathrm{~g}, 0.49 \mathrm{mmol}$, $48 \%$ ) , mp 198-200 ${ }^{\circ} \mathrm{C}$.

${ }^{1} \mathrm{H}$ NMR $\left(\mathrm{CDCl}_{3}\right) \delta 1.59\left(\mathrm{~d}, \mathrm{~J}=6.4 \mathrm{~Hz}, 3 \mathrm{H}, \mathrm{CH}_{3}\right), 2.30-2.48\left(\mathrm{~m}, 4 \mathrm{H}, \mathrm{H}^{1} / \mathrm{H}^{2}\right), 3.34(\mathrm{dd}, \mathrm{J}=14.4,2.8$ $\left.\mathrm{Hz}, 1 \mathrm{H}, \mathrm{H}^{6 \beta}\right), 3.47$ (dd, J=14.4, $11.1 \mathrm{~Hz}, 1 \mathrm{H}, \mathrm{H}^{6 \alpha}$ ), 4.32 (br s, $1 \mathrm{H}, \mathrm{H}^{4 \mathrm{a}}$ ), 5.00 (ddq, $\mathrm{J}=11.1,2.8$, 6.4 Hz, $\left.1 \mathrm{H}, \mathrm{H}^{7}\right), 5.83-5.91\left(\mathrm{~m}, 1 \mathrm{H}, \mathrm{H}^{4}\right), 6.33-6.42\left(\mathrm{~m}, 1 \mathrm{H}, \mathrm{H}^{3}\right) ;{ }^{13} \mathrm{C} \mathrm{NMR}\left(\mathrm{CDCl}_{3}\right) \delta 20.2,23.2$, 28.5, 54.4, 64.3, 68.0, 74.9, 112.9, 136.1, 172.1; X-ray crystal structure reported in ref. 21c.

NOE $(500 \mathrm{MHz})$ : irradiation of $\mathrm{H}^{4 a}$ resulted in strong enhancement of $\mathrm{H}^{4}$ and $\mathrm{H}^{6 \alpha}$; irradiation of $\mathrm{H}^{7}$ resulted in enhancement of $\mathrm{CH}_{3}$ and $\mathrm{H}^{6 \beta}$.

The minor product $\mathbf{1 6 g}$ partially decomposed on the silica column to produce dehydrobromination product $\mathbf{1 8 g}\left(\mathrm{R}_{\mathrm{f}}=0.16\right)$.

(4aSR,7SR,9aRS)-9a-Bromo-7-methyl-5,5-dioxo-1,2,4a,9a-tetrahydro-8-oxa-5 $\lambda^{6}$ thiabenzocycloheptan-9-one (16g).

A very small analytical sample of the title compound $\mathbf{1 6} \mathrm{g}$ for X-ray diffraction analysis and melting point determination was obtained as a white crystalline solid (mp 198-200 ${ }^{\circ} \mathrm{C}$, decomposes) by slow evaporation of $\mathrm{CDCl}_{3}$ from an NMR tube containing the ca $100 / 15$ mixture of $16 \mathrm{~g}$ and $\mathbf{1 5 g}$, obtained by procedure 1.18.7. The ${ }^{1} \mathrm{H}$ and ${ }^{13} \mathrm{C}$ NMR data of the title compound $16 \mathrm{~g}$ was assigned from this mixture.

${ }^{1} \mathrm{H} \mathrm{NMR}\left(\mathrm{CDCl}_{3}\right) \delta 1.59\left(\mathrm{~d}, \mathrm{~J}=6.4 \mathrm{~Hz}, 3 \mathrm{H}, \mathrm{CH}_{3}\right), 2.26-2.48\left(\mathrm{~m}, 3 \mathrm{H}, \mathrm{H}^{1} / \mathrm{H}^{2}\right), 2.53-2.63(\mathrm{~m}, 1 \mathrm{H}$, $\left.\mathrm{H}^{1} / \mathrm{H}^{2}\right), 3.43\left(\mathrm{dd}, \mathrm{J}=14.5,8.5 \mathrm{~Hz}, 1 \mathrm{H}, \mathrm{H}^{6 \beta}\right), 3.58\left(\mathrm{dd}, \mathrm{J}=14.5,3.8 \mathrm{~Hz}, 1 \mathrm{H}, \mathrm{H}^{6 \alpha}\right), 4.44(\mathrm{br} \mathrm{s}, 1 \mathrm{H}$, $\left.\mathrm{H}^{4 \mathrm{a}}\right), 5.41\left(\mathrm{ddq}, \mathrm{J}=8.6,3.8,6.3 \mathrm{~Hz}, 1 \mathrm{H}, \mathrm{H}^{7}\right), 5.78-5.87\left(\mathrm{~m}, 1 \mathrm{H}, \mathrm{H}^{4}\right), 6.35-6.45\left(\mathrm{~m}, 1 \mathrm{H}, \mathrm{H}^{3}\right) ;{ }^{13} \mathrm{C}$ $\operatorname{NMR}\left(\mathrm{CDCl}_{3}\right) \delta 20.3,22.7,31.4,57.5,60.9,66.5,69.9,112.6,135.8,167.7$; X-ray crystal structure reported in ref. $21 \mathrm{~d}$.

NOE $(500 \mathrm{MHz})$ : irradiation of $\mathrm{H}^{4 a}$ resulted in strong enhancement of $\mathrm{H}^{4}, \mathrm{H}^{7}$ and in enhancement of $\mathrm{H}^{6 \alpha}$; irradiation of $\mathrm{H}^{7}$ resulted in strong enhancement of $\mathrm{H}^{4 \mathrm{a}}$ and enhancement of $\mathrm{H}^{6 \alpha}$ and $\mathrm{CH}_{3}$. 
7-Methyl-5,5-dioxo-1,2-dihydro-8-oxa-5 $\lambda^{6}$-thiabenzocycloheptan-9-one (18g).

A small sample of the title compound $\mathbf{1 8} \mathbf{g}$ was collected after the column chromatography in procedure 1.18.7.

${ }^{1} \mathrm{H} \mathrm{NMR}\left(\mathrm{CDCl}_{3}\right) \delta 1.44\left(\mathrm{~d}, \mathrm{~J}=6.3 \mathrm{~Hz}, 1 \mathrm{H}, \mathrm{CH}_{3}\right), 2.26-2.65\left(\mathrm{~m}, 3 \mathrm{H}, \mathrm{H}^{1} / \mathrm{H}^{2}\right), 2.81-2.98(\mathrm{~m}, 1 \mathrm{H}$, $\left.\mathrm{H}^{1} / \mathrm{H}^{2}\right), 3.62\left(\mathrm{dd}, \mathrm{J}=14.1,10.7 \mathrm{~Hz}, 1 \mathrm{H}, \mathrm{H}^{6 \alpha}\right), 3.58\left(\mathrm{dd}, \mathrm{J}=14.1,3.5 \mathrm{~Hz}, 1 \mathrm{H}, \mathrm{H}^{6 \beta}\right), 5.41(\mathrm{ddq}$, $\left.\mathrm{J}=10.6,3.7,6.3 \mathrm{~Hz}, 1 \mathrm{H}, \mathrm{H}^{7}\right), 5.37\left(\mathrm{ddd}, \mathrm{J}=9.8,5.1,4.3 \mathrm{~Hz}, 1 \mathrm{H}, \mathrm{H}^{4}\right), 6.49(\mathrm{dt}, \mathrm{J}=9.8,1.8 \mathrm{~Hz}, 1 \mathrm{H}$, $\left.\mathrm{H}^{3}\right)$.

1.18.8. (4aSR,7SR,9aRS)-9a-Bromo-7-phenyl-5,5-dioxo-1,2,4a,9a-tetrahydro-8-oxa-5 $\lambda^{6}$ thiabenzocycloheptan-9-one (15h).

$\beta$-Acyloxy sulfone $\mathbf{1 4 h}(0.35 \mathrm{~g}, 0.94 \mathrm{mmol})$ was heated in toluene $(94 \mathrm{~mL}, 0.010 \mathrm{M})$ at $127{ }^{\circ} \mathrm{C}$ for $21 \mathrm{~h}$. Column chromatography (EtOAc/Hexanes, 1/5) gave the title compound $\mathbf{1 5 h}$ as a white solid $\left(0.22 \mathrm{~g}, 0.59 \mathrm{mmol}, 63 \%, \mathrm{R}_{\mathrm{f}}=0.28\right), \mathrm{mp} 212-214{ }^{\circ} \mathrm{C}$. The minor endo-product $\mathbf{1 6 h}$ decomposed on the silica column.

${ }^{1} \mathrm{H} \mathrm{NMR}\left(\mathrm{CDCl}_{3}\right) \delta$ 2.36-2.51 (m, 4H, H$\left.{ }^{1} / \mathrm{H}^{2}\right), 3.58\left(\mathrm{dd}, \mathrm{J}=14.5,2.8 \mathrm{~Hz}, 1 \mathrm{H}, \mathrm{H}^{6 \beta}\right), 3.80(\mathrm{dd}$, $\left.\mathrm{J}=14.5,11.5 \mathrm{~Hz}, 1 \mathrm{H}, \mathrm{H}^{6 \alpha}\right), 4.47\left(\right.$ br s, $\left.1 \mathrm{H}, \mathrm{H}^{4 \mathrm{a}}\right), 5.87\left(\mathrm{dd}, \mathrm{J}=11.5,2.8 \mathrm{~Hz}, 1 \mathrm{H}, \mathrm{H}^{7}\right), 5.87-5.96(\mathrm{~m}$, $\left.1 \mathrm{H}, \mathrm{H}^{4}\right), 6.36-6.45\left(\mathrm{~m}, 1 \mathrm{H}, \mathrm{H}^{3}\right), 7.37-7.47(\mathrm{~m}, 5 \mathrm{H}, \mathrm{Ph}) ;{ }^{13} \mathrm{C} \mathrm{NMR}\left(\mathrm{CDCl}_{3}\right) \delta 23.2,28.6,55.2$, $64.0,68.4,78.8,112.8,126.1,129.3,129.6,135.3,136.3,171.8$. 


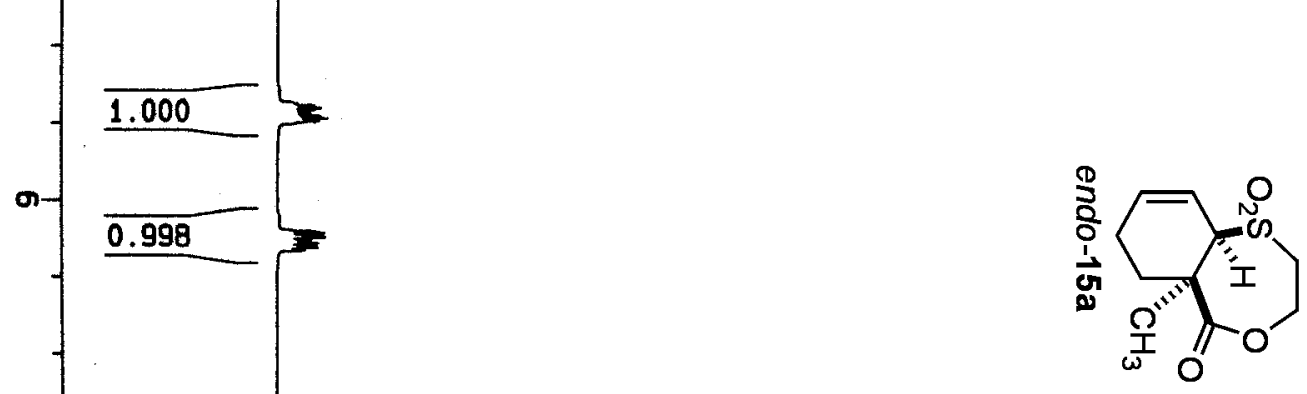




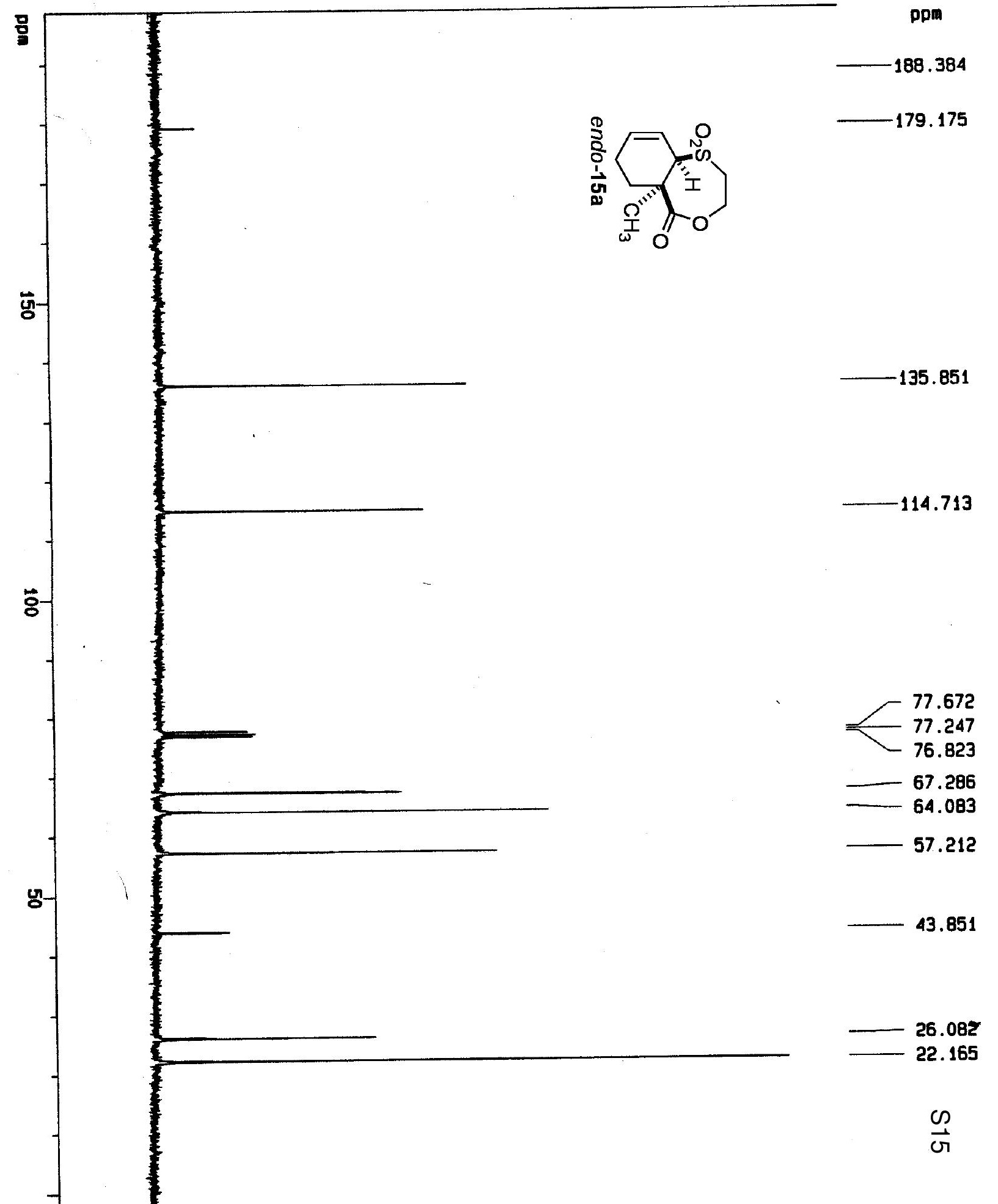




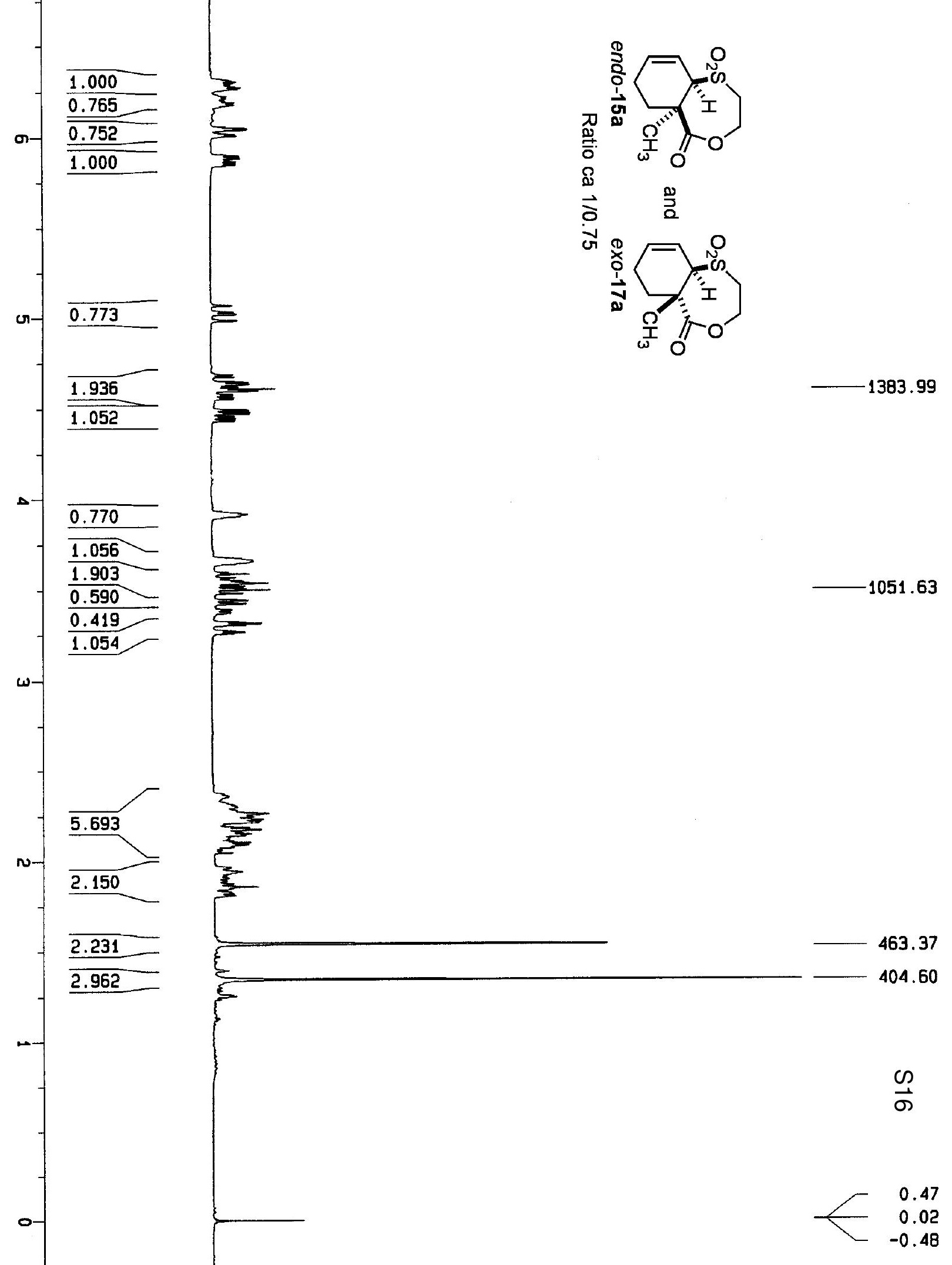




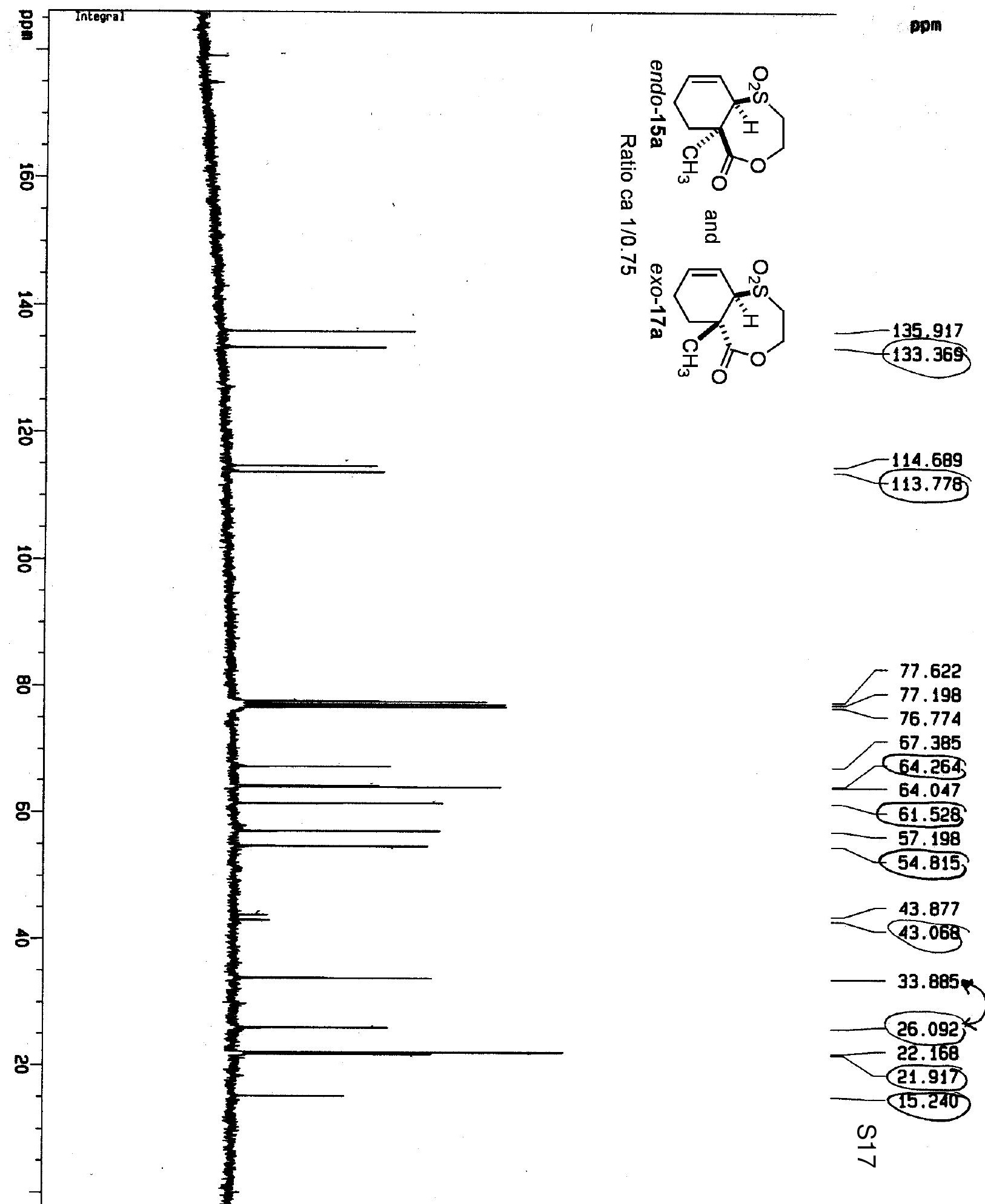




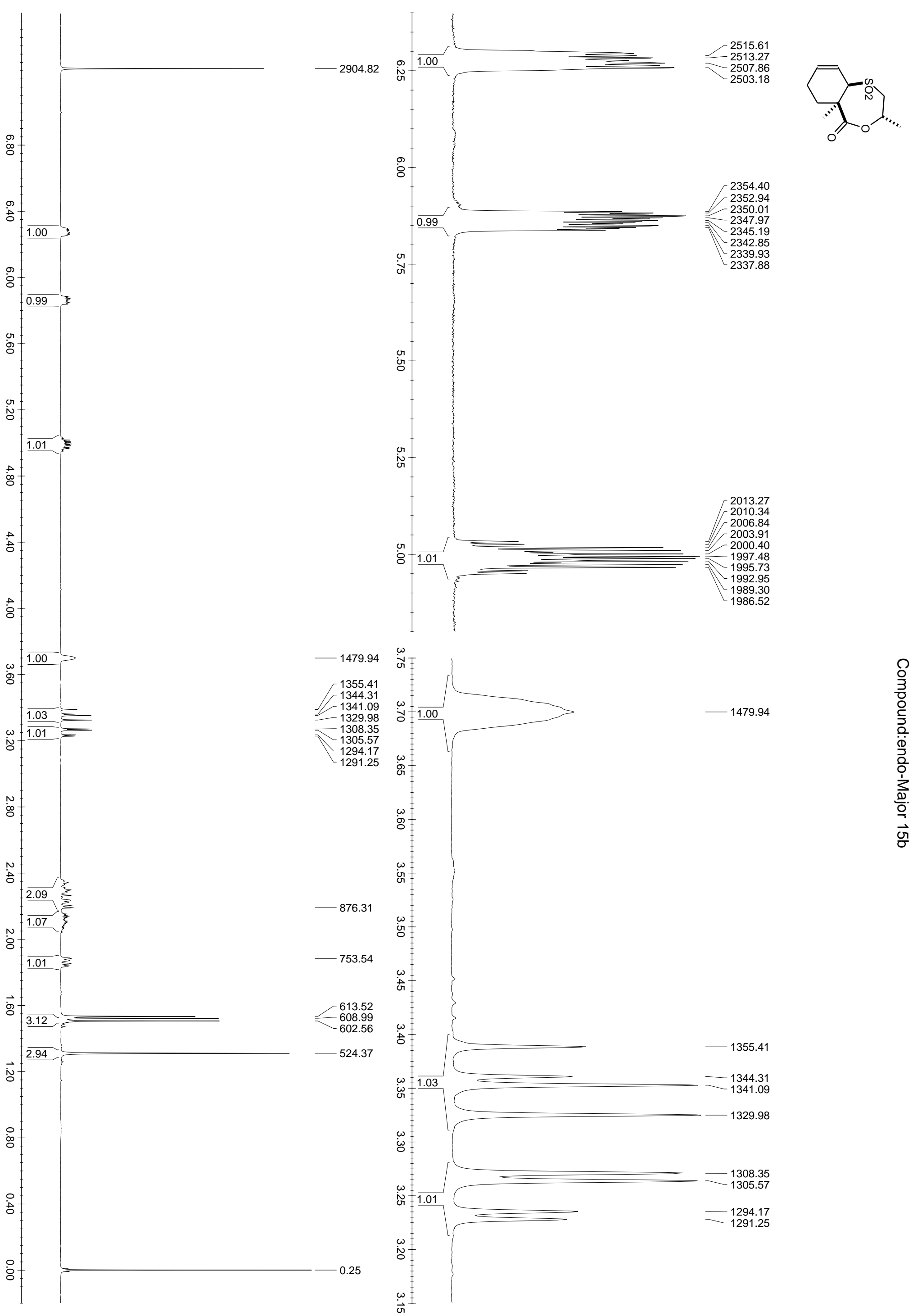




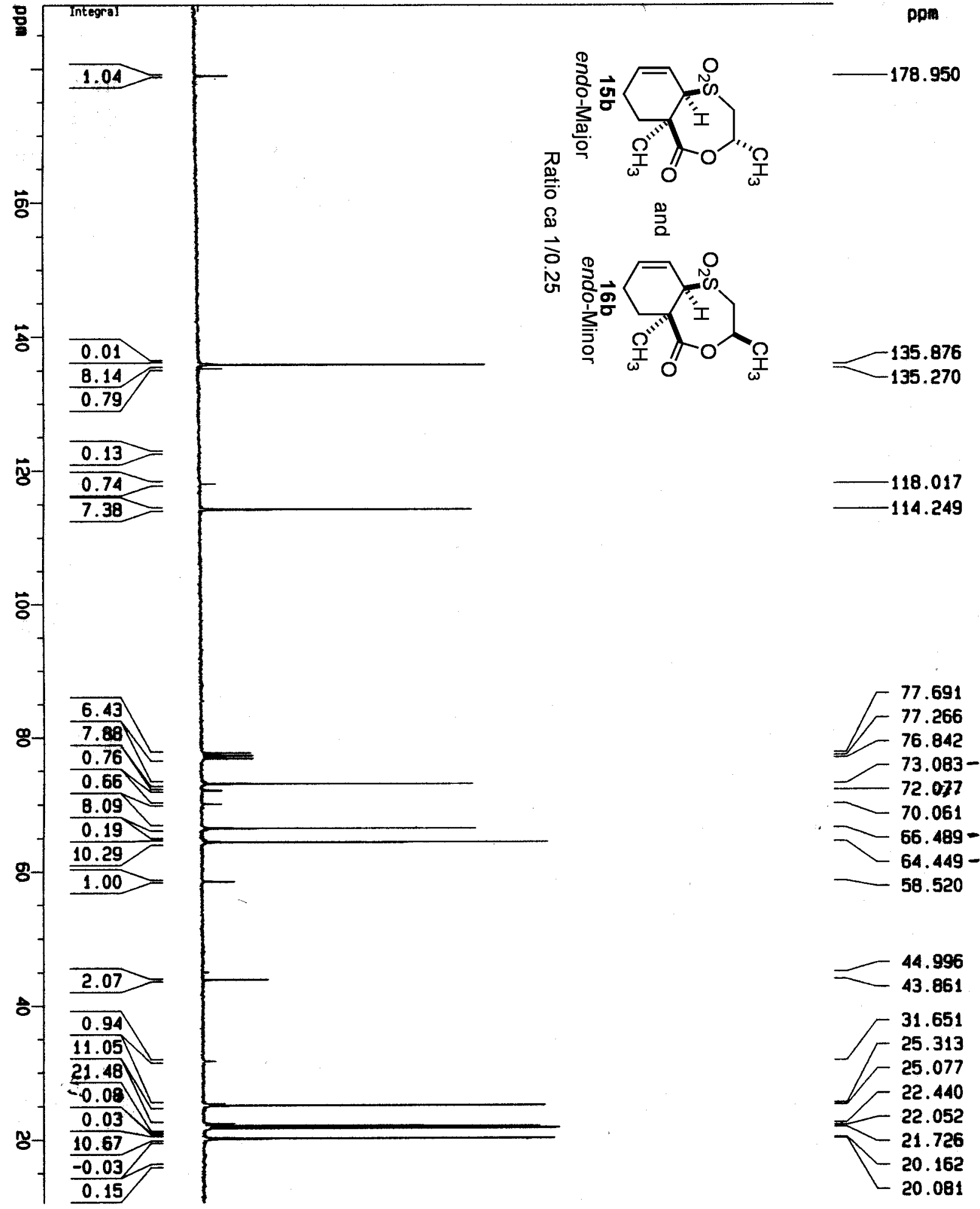



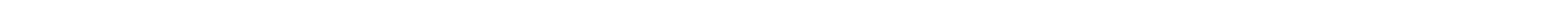

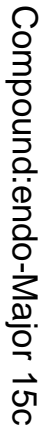




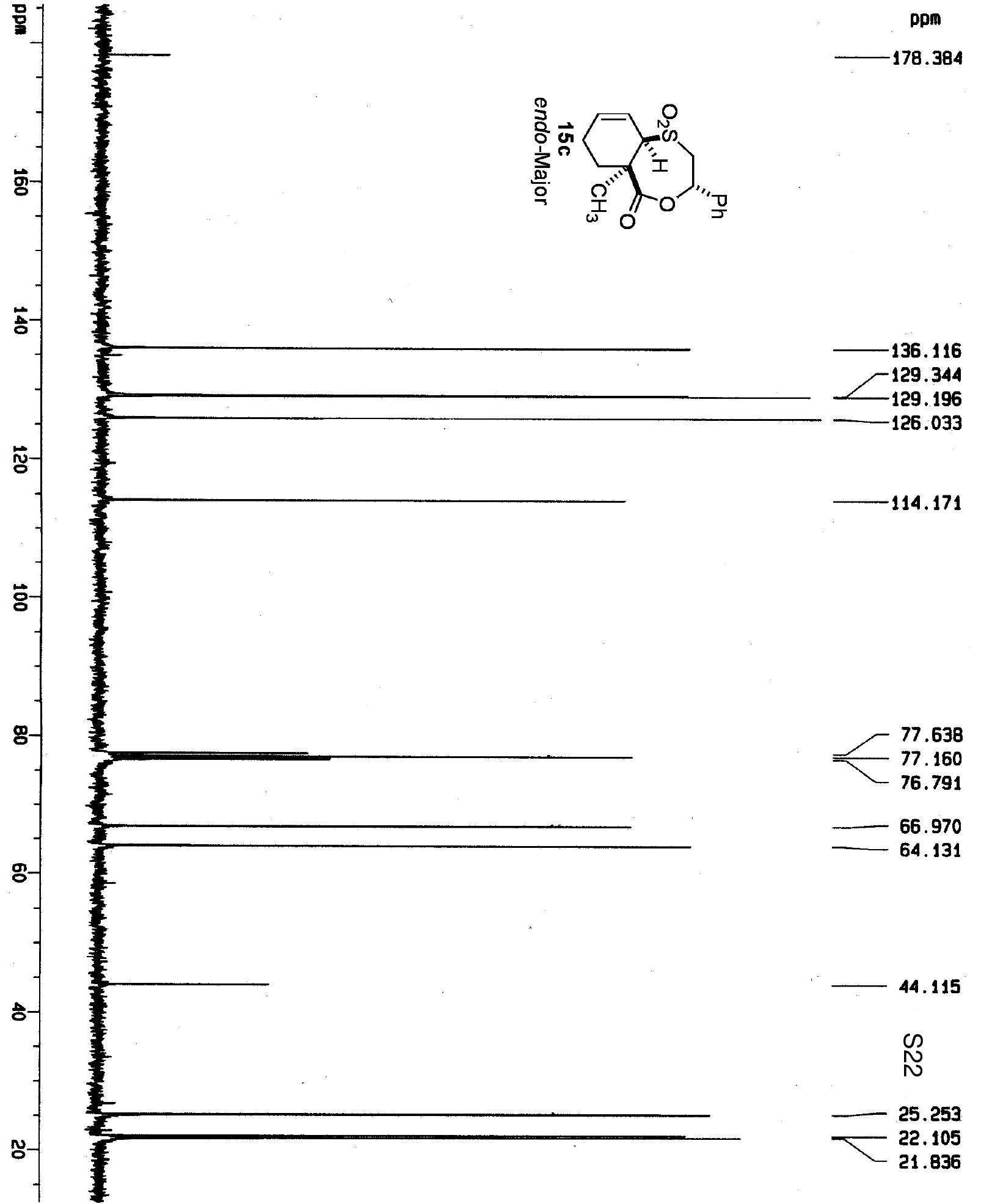



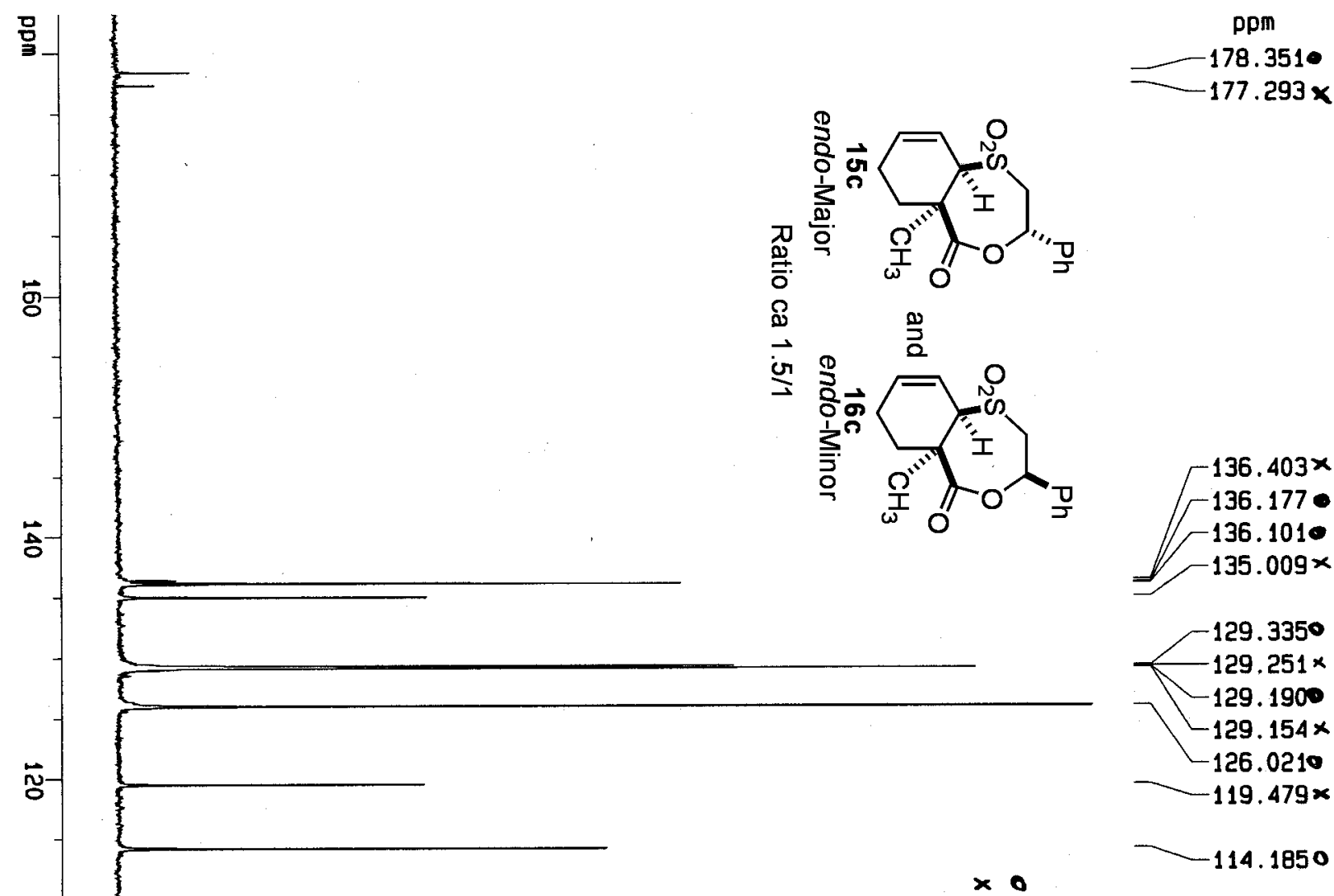

के

홍
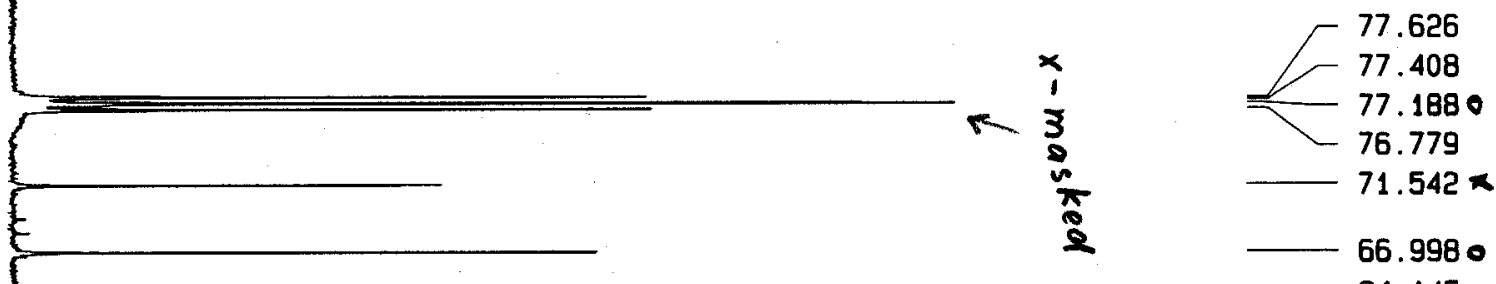

$64.145 \circ$

$g$

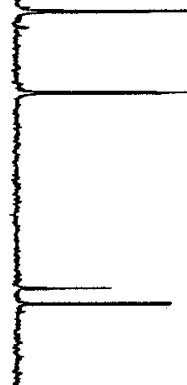

$58.597 x$

$129.351 \times$

129.1900

$-129.154 x$

126.0210

$-119.479 x$

114.1850

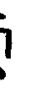




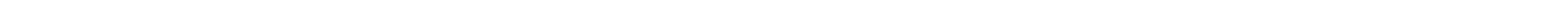




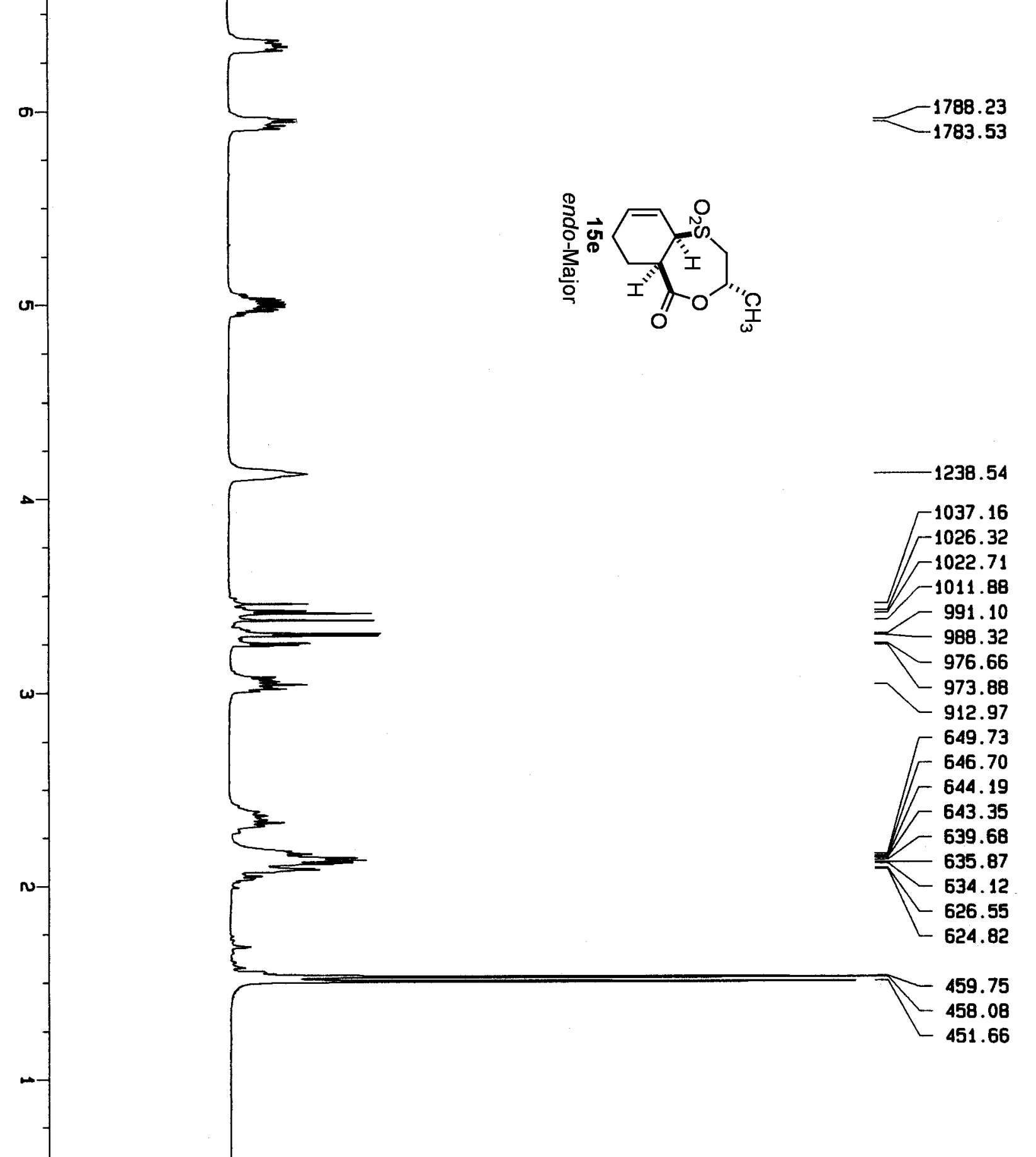




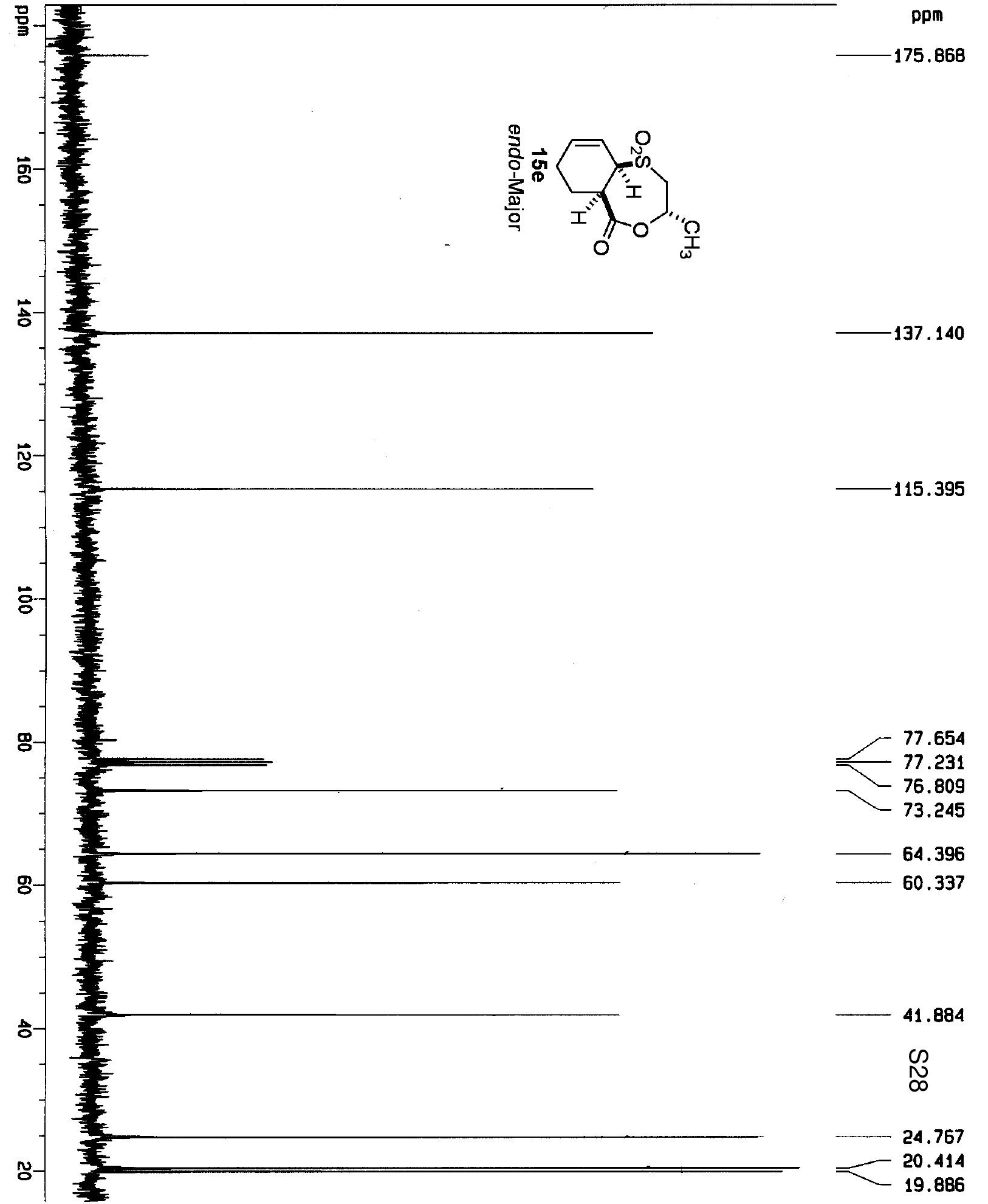





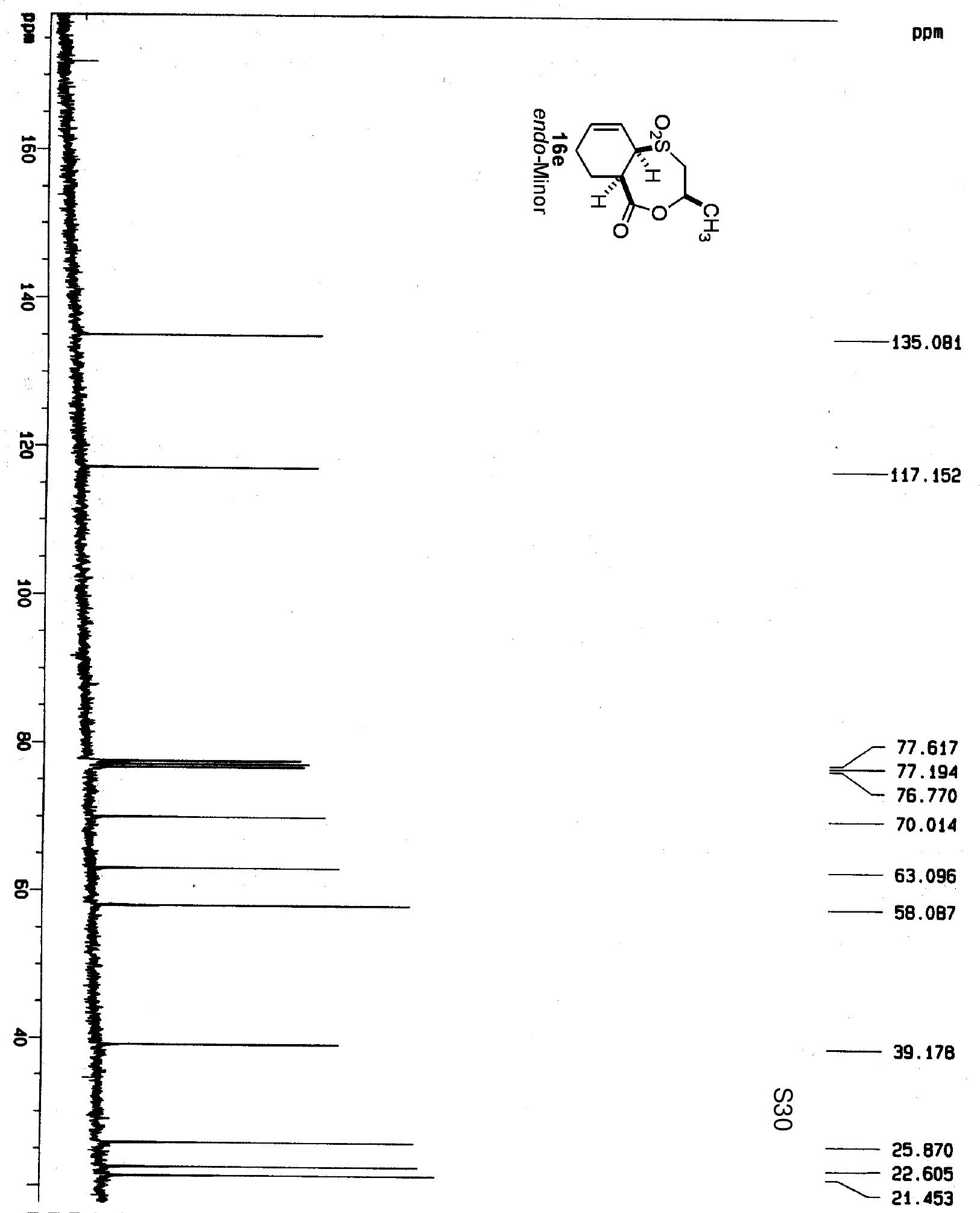




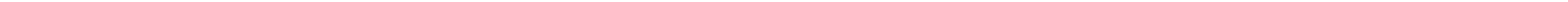




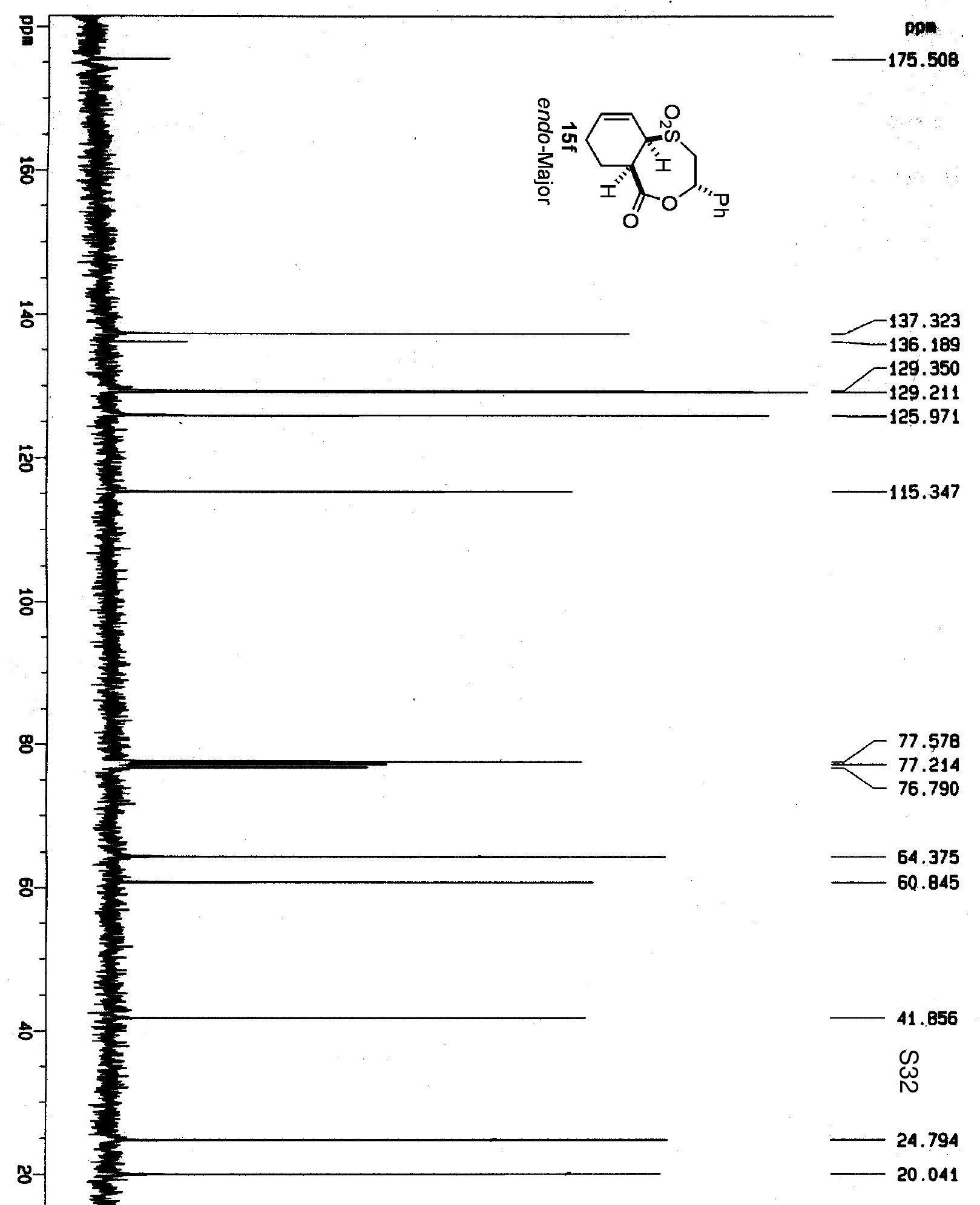




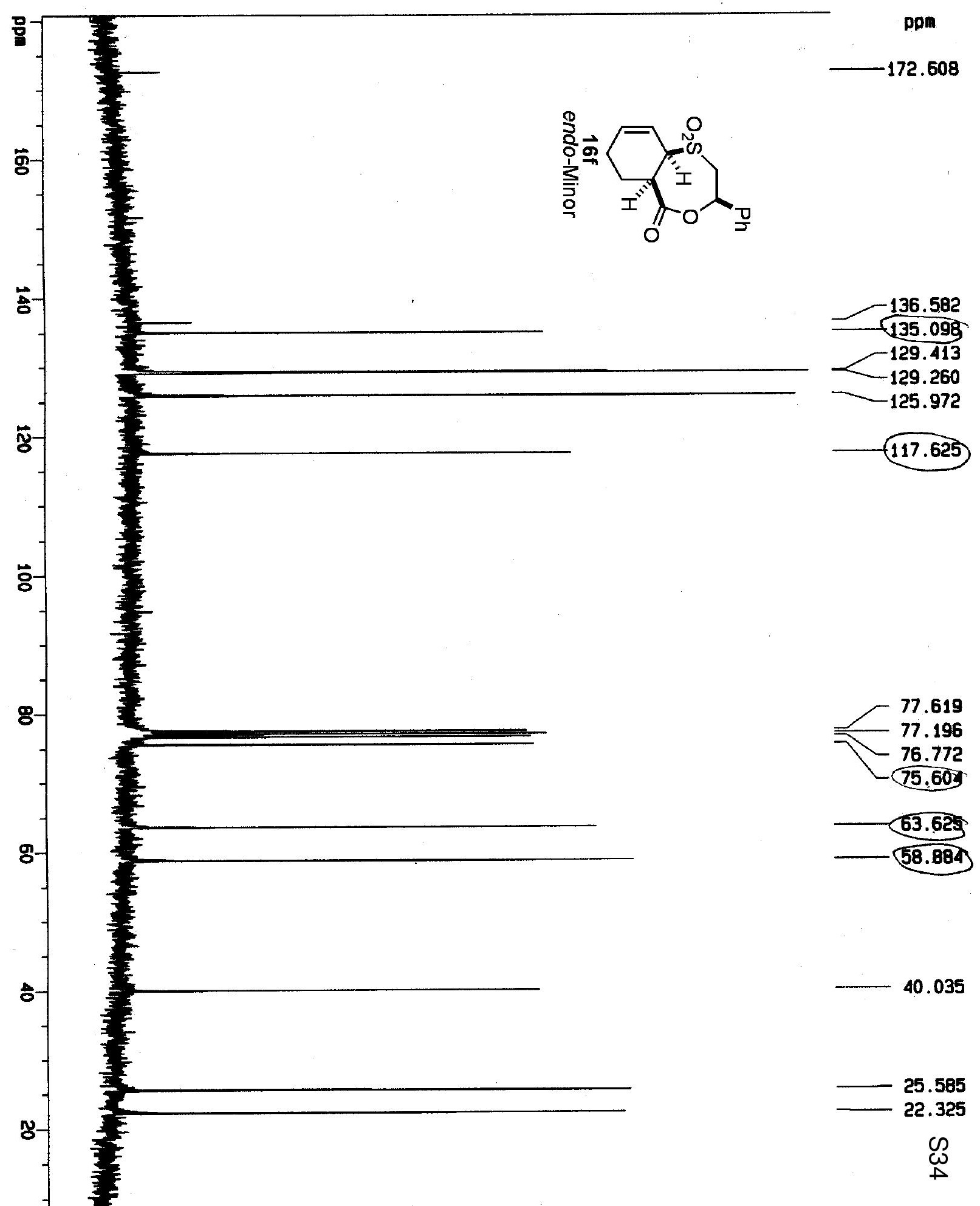




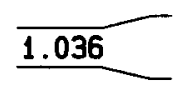

$=$

o-
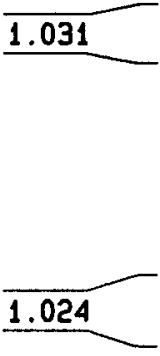

1.000
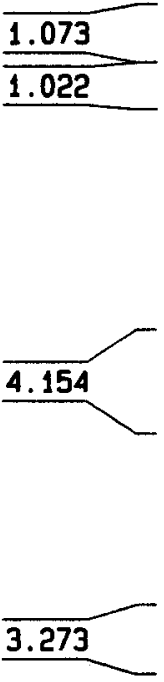

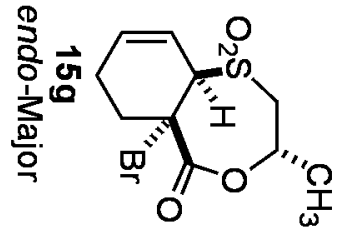

W

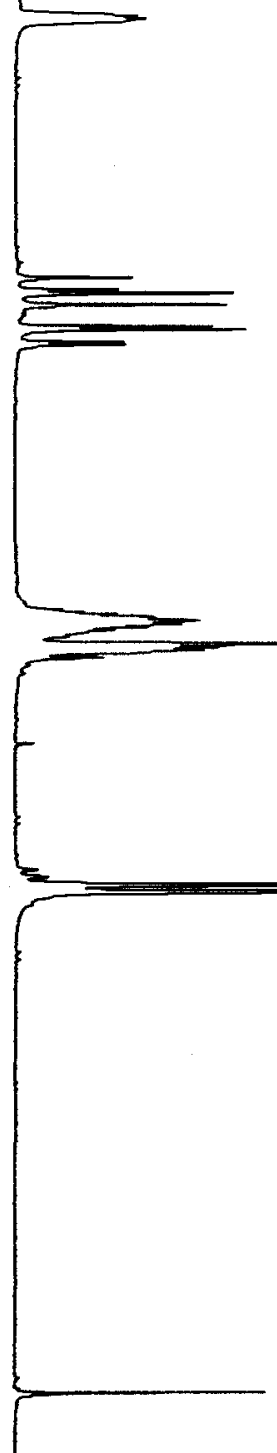

1297.02 $-1053.67$ $-1042.56$ 1039.25 1028.15 1007.20 1006.87 1004.39 992.79 992.43 989.97 736.05 734.03 732.22 730.51 726.11 723.55 721.04 707.68 705.16 $704: 72$ 701.83 481.74 479.49 475.32 473.64 473.06 


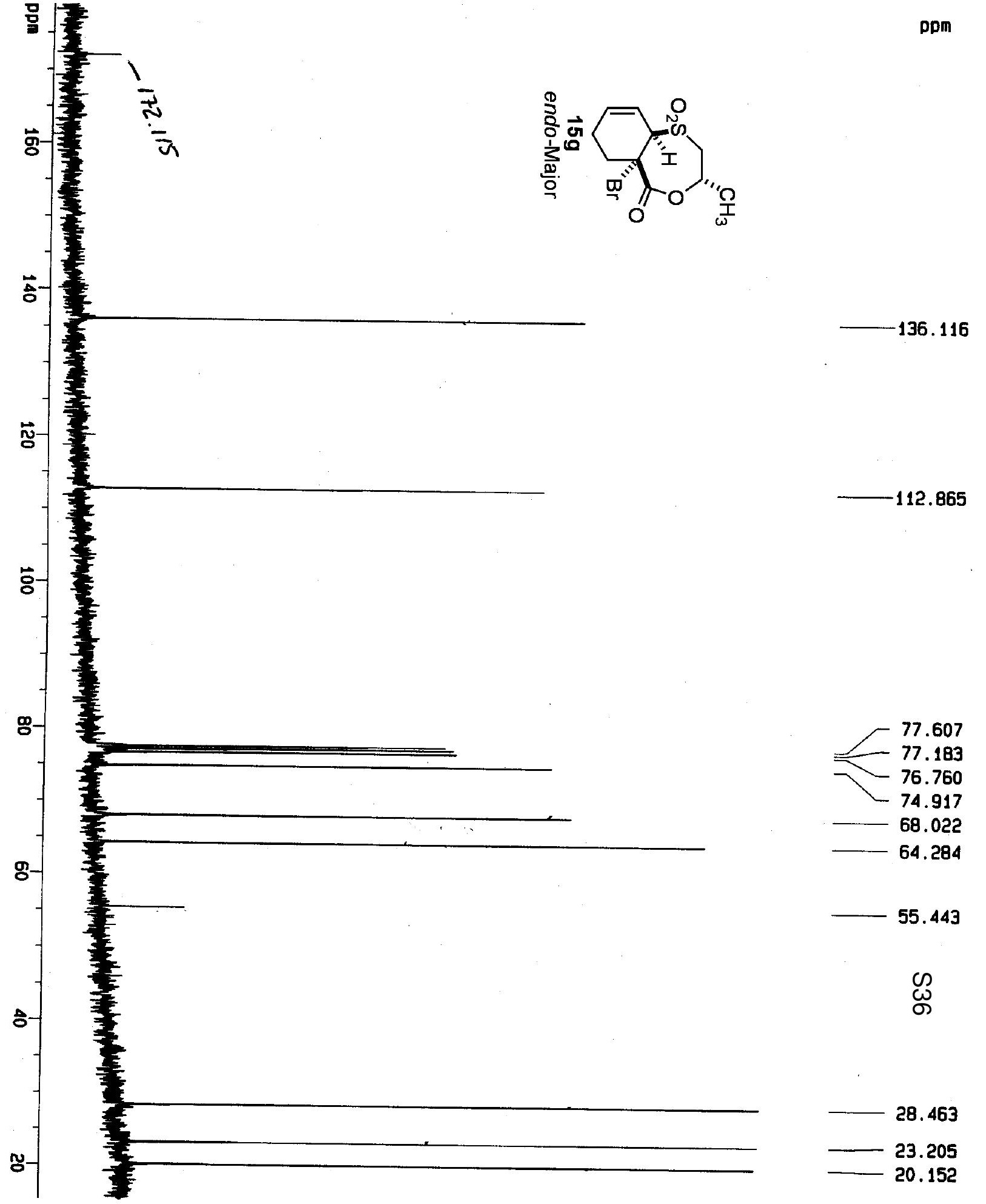




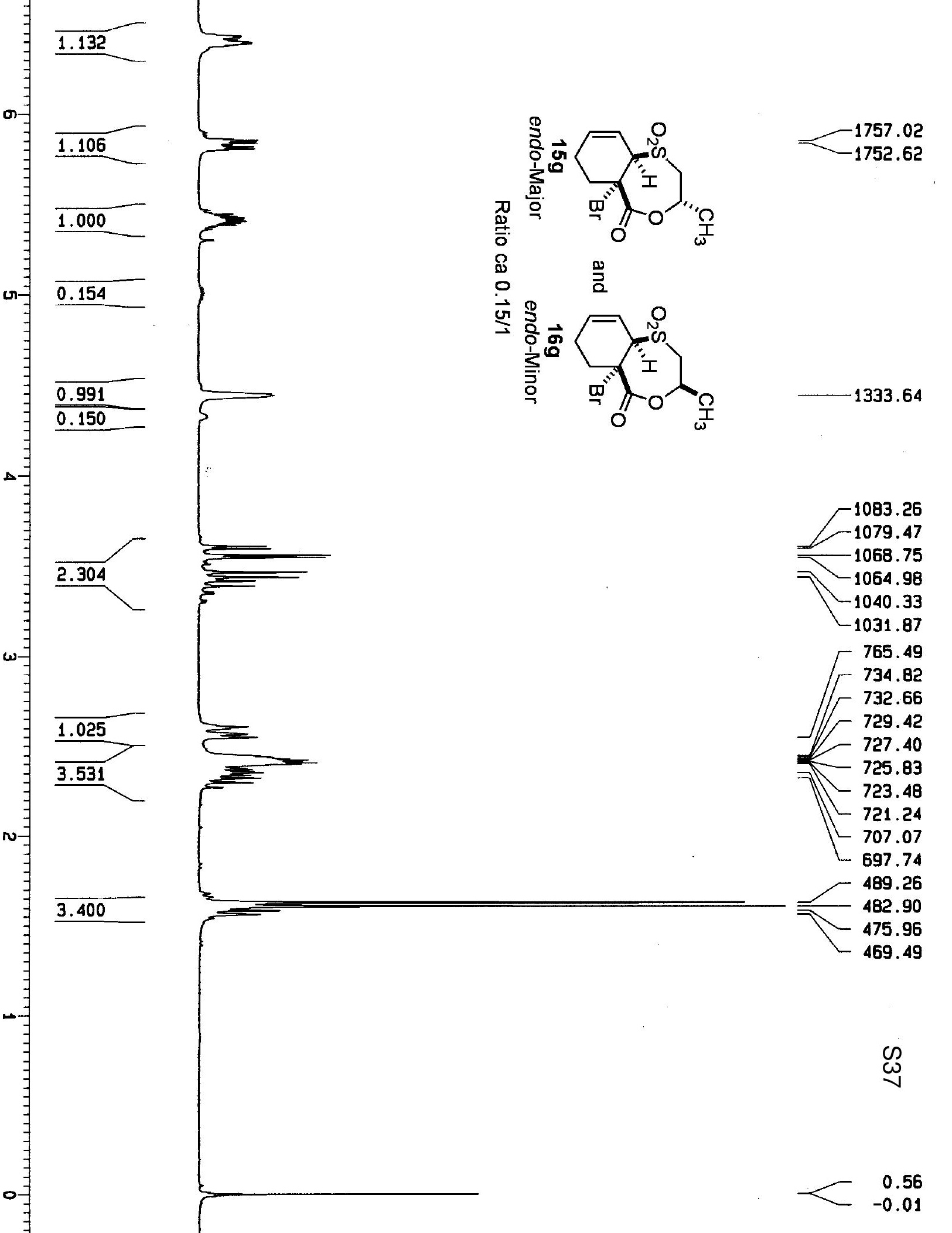




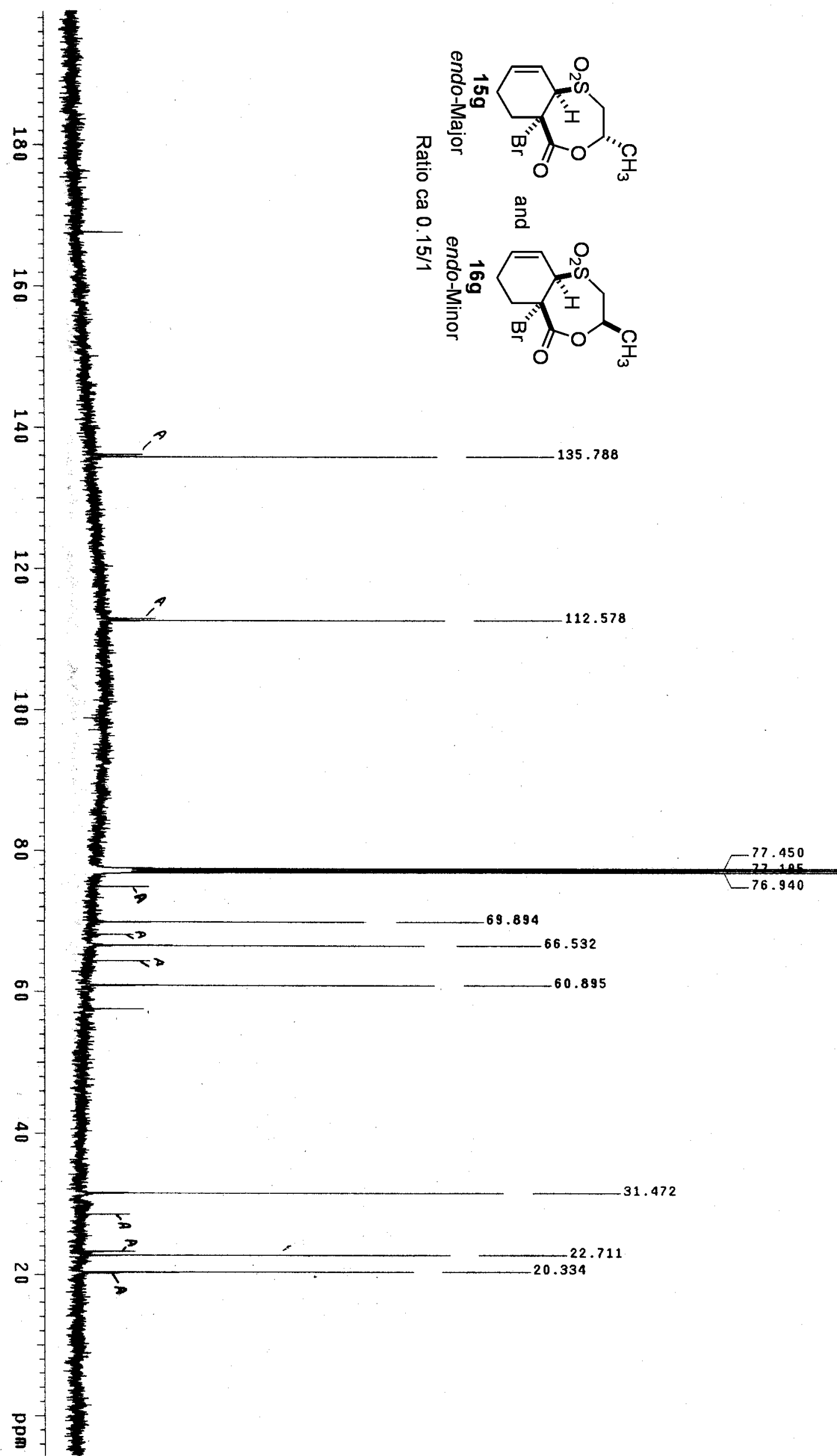




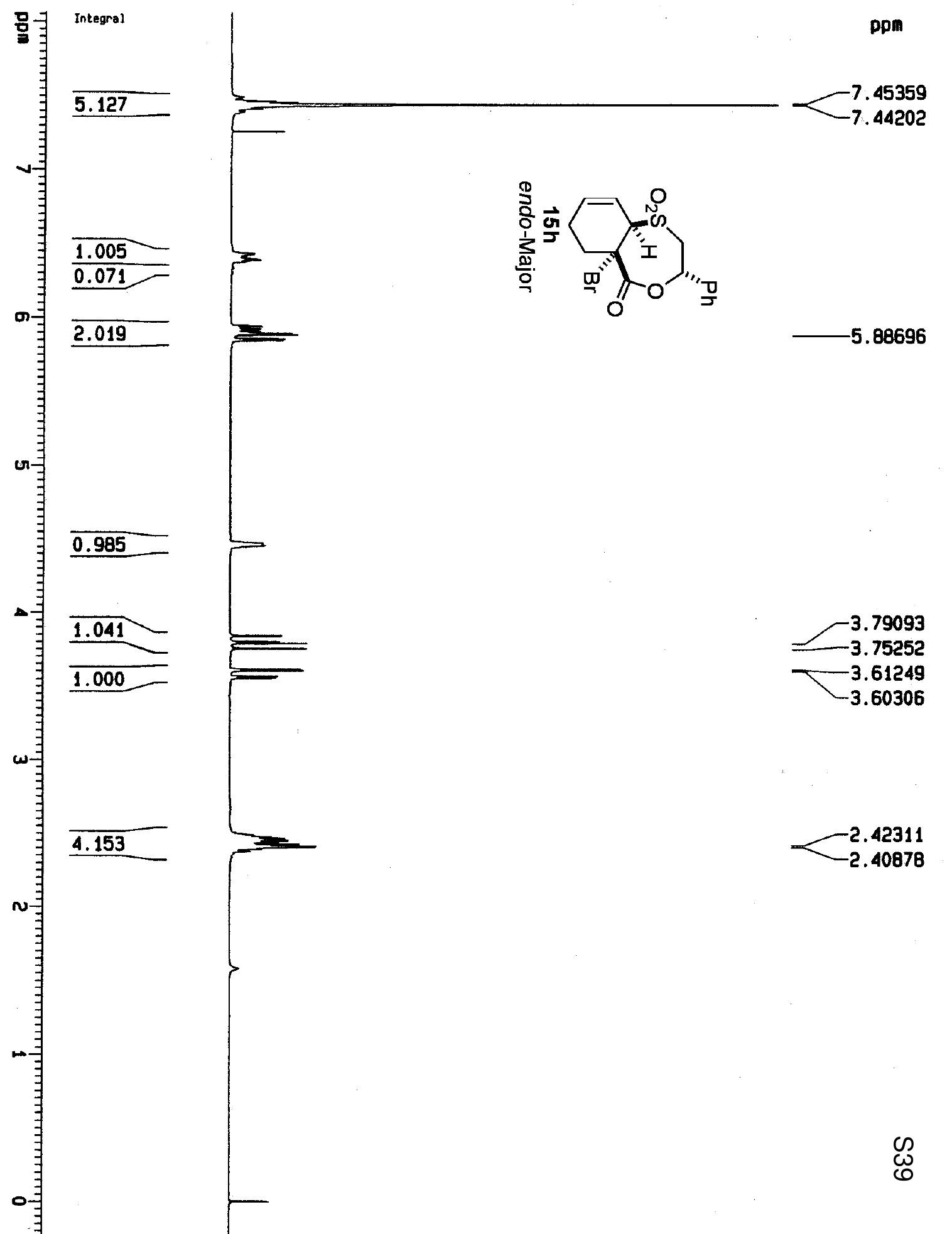


